\title{
Normative pathways in the functional connectome
}

\author{
Matthew LemingA*, Li SuA,B, Shayanti ChattopadhyayA, John SucklingA \\ ADepartment of Psychiatry, University of Cambridge, Cambridge, UK \\ BChina-UK Centre for Cognition and Ageing Research, Faculty of Psychology, \\ Southwest University, Chongqing, China \\ *Correspondence: ml784@cam.ac.uk \\ Herchel Smith Bldg, Robinson Way, Cambridge, UK CB2 0SZ
}

August 2018 


\section{Abstract}

Functional connectivity is frequently derived from fMRI data to reduce a complex image of the brain to a graph, or "functional connectome". Often shortest-path algorithms are used to characterize and compare functional connectomes. Previous work on the identification and measurement of semi-metric (shortest circuitous) pathways in the functional connectome has discovered cross-sectional differences in major depressive disorder (MDD), autism spectrum disorder (ASD), and Alzheimer's disease. However, while measurements of shortest path length have been analyzed in functional connectomes, less work has been done to investigate the composition of the pathways themselves, or whether the edges composing pathways differ between individuals. Developments in this area would help us understand how pathways might be organized in mental disorders, and if a consistent pattern can be found. Furthermore, studies in structural brain connectivity and other real-world graphs suggest that shortest pathways may not be as important in functional connectivity studies as previously assumed. In light of this, we present a novel measurement of the consistency of pathways across functional connectomes, and an algorithm for improvement by selecting the most frequently occurring "normative pathways" from the k shortest paths, instead of just the shortest path. We also look at this algorithm's effect on various graph measurements, using randomized matrix simulations to support the efficacy of this method and demonstrate our algorithm on the resting-state fMRI (rs-fMRI) of a group of 34 adolescent control participants. Additionally, a comparison of normative pathways is made with a group of 82 age-matched participants, diagnosed with MDD, and in doing so we find the normative pathways that are most disrupted. Our results, which are carried out with estimates of connectivity derived from correlation, partial correlation, and normalized mutual information connectomes, suggest disruption to the default mode, affective, and ventral attention networks. Normative pathways, especially with partial correlation, make greater use of critical anatomical pathways through the striatum, cingulum, and the cerebellum. In summary, MDD is characterized by a disruption of normative pathways of the ventral attention network, increases in alternative pathways in the frontoparietal network in MDD, and a mixture of both in the default mode network. Additionally, within- and between-groups findings depend on the estimate of connectivity.

Keywords: Functional connectivity; major depressive disorder; pathways; adolescent depression; graph theory; resting-state fMRI

\section{Introduction}

\subsection{Resting State fMRI and Connectomics}

Functional Magnetic Resonance Imaging (fMRI) acquires temporal information on blood-oxygen level dependent (BOLD) signals from the human brain. Functional connectomics (Friston et al., 1993) reduces the dimensionality of these datasets to graphs (composed of nodes, representing brain areas, connected by edges) that illustrate the relationships between areas of the brain. Graph theory estimates the qualities of brain organization with measures such as centrality (or "hubness")(Sporns et al., 2007; Joyce et al., 2010; Lohmann et al., 2010; Rubinov and Sporns, 2010; Tomasi and Volkow, 2010, 2011; Zuo et al., 2011) and community structure (or "modularity")(Traag and Bruggeman, 2009; Mucha et al., 2010; Bassett et al., 2013; Sporns and Betzel, 2016). In general, the functional connectome is characterized by high complexity (Sporns et al., 2000; Sporns, 2006), high efficiency (Buzsaki et al., 2004), global and local synchronizability(Masuda and Aihara, 2004), and high levels of clustering with short path lengths(Hilgetag et al., 2000; Stephan et al., 2000; Bassett and Bullmore, 2006), indicating a small-world architecture(Milgram, 1967; Watts and Strogatz, 1998).

\subsection{Path analysis of connectomes}

Studies of average shortest path length (Gong et al., 2009; Yan et al., 2011; Lynall et al., 2010; Betzel et al., 2014) and its inverse, graph efficiency (Latora and Marchiori, 2001), have been conducted on both binarised functional (Bassett and Bullmore, 2006; Sporns et al., 2007; Wang et al., 2009; Lynall et al., 2010) and structural connectomes (Achard and Bullmore, 2007; Gong et al., 2009; Yan et al., 2011). Related to these measures are "rich clubs" (van den Heuvel and Sporns, 2011; van den Heuvel et al., 2012) that measure the tendency of nodes with high degree to be more densely connected amongst themselves than with other nodes of the connectome, which has implications for which nodes tend to be the most utilized in pathways. Like the 
functional connectome, an efficient, small-world structure has been shown to characterize the structural connectome(Hilgetag et al., 2000; Sporns and Zwi, 2004; Gong et al., 2009; Yan et al., 2011). Shortest-pathbased node centrality measurements (such as betweenness (Freeman, 1977), regional efficiency(Latora and Marchiori, 2001; Achard and Bullmore, 2007), and closeness (Freeman, 1979)) are outlined and discussed in Sporns et al. (2007), Joyce et al. (2010), Zuo et al. (2011), and Rubinov and Sporns (2010).

The majority of connectomic analyses assume the importance of the shortest pathway, even though real- world networks often do not have knowledge of their own global structure (Boguña et al., 2009; Abdelnour et al., 2014; Goñi et al., 2013b), and so in practice, the shortest pathway is unlikely to utilized by prior planning (da Fontoura Costa and Travieso, 2007; Serrano et al., 2007; Estrada and Hatano, 2008). Studies of structural connectivity have investigated the relationships between two nodes other than the shortest pathway, such as path ensembles derived from the $k$ shortest pathways (Avena-Koenigsberger et al., 2017), maximum flow (Yoo et al., 2015), and robustness (Kaiser et al., 2007). Furthermore, the structural con- nectome is both a predictor and a constraint for neural communication across the functional connectome (Passingham et al., 2002; Galán, 2008; Honey et al., 2009; Hermundstad et al., 2013; Park and Friston, 2013; Goñi et al., 2013a; Betzel et al., 2014; Mišić et al., 2015), and thus we hypothesize that alternatives to the shortest pathway provide a richer description of the topology of the functional connectome.

\subsection{Previous work on semimetric analysis of functional connectomes}

Functional connectomes are represented, in the case of Pearson correlations, as a positive semi-definite matrix with values on $[-1,1]$ or, in the case of alternative measurements like normalized mutual information, as a matrix with values on $[0,1]$. It is often the case that path finding is performed after thresholding of edges to generate a binary graph with nodes defined as voxels (van den Heuvel et al., 2009) or regional parcels of the brain (Sporns et al., 2000; Stephan et al., 2000; Bassett and Bullmore, 2006). More recently, however, path finding on unthresholded functional connectomes has been undertaken (Rocha, 2002; Cao et al., 2014; Simas and Rocha, 2014; Simas et al., 2015; Suckling et al., 2015) by inverting them from a proximity graph to a distance graph, which is embedded in a semimetric space (see Methods; Figure 1; Appendix A).

Previous studies have shown both sensitivity and specificity in differentiating control participants from individuals with autism spectrum disorder (ASD) and major depressive disorder (MDD) (Simas et al., 2015) using the proportion of edges in semimetric distance space with a shorter indirect path: the semimetric percentage. Additionally, Suckling et al. (2015) used a similar semimetric analysis to classify patients with Alzheimer's Disease (AD). Although successful in distinguishing alterations in brain functional organization, these semimetric approaches rendered scalar measurements for large regions of the brain without investigating the origins of the changes, and in particular the edge composition of the constituent pathways.

Whilst there may be a difference in the proportion of shortest paths between two nodes that are indirect, there has not yet been a characterization of the routing of the shortest indirect paths, or their consistency of routing through particular areas of the brain. Furthermore, if the shortest indirect path among individuals is inconsistent, is there a second, third, or kth shortest pathway that consistently connects two areas? And do indirect paths differ in patients with mental health disorders; for example, MDD, as compared to healthy individuals?

Many psychiatric and neurological (Delbeuck et al., 2003) disorders are now being characterized from the perspective of altered or disrupted connectivity. Functional plasticity is central to the development and ageing of the brain (Anderson and Thomason, 2013), its response to injury (Anderson et al., 2005), and neurodegeneration (Greenwood, 2007). Thus, an expansive analysis of the constellation of shortest paths that route information through complex brain networks is key to a deeper understanding of the information contained within the functional connectome. Below, we identify and analyze the normative pathways, which refer to a set of the most consistently occurring of the $\mathrm{k}$ shortest pathways across a group of connectomes. 


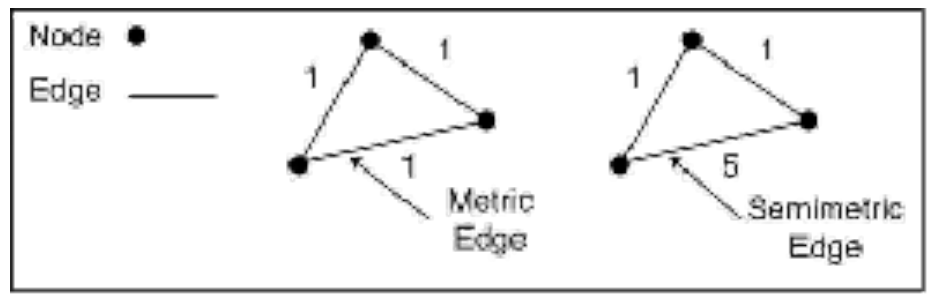

\section{Binary/unweighted graph}

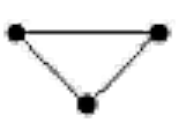

Weighted graphs

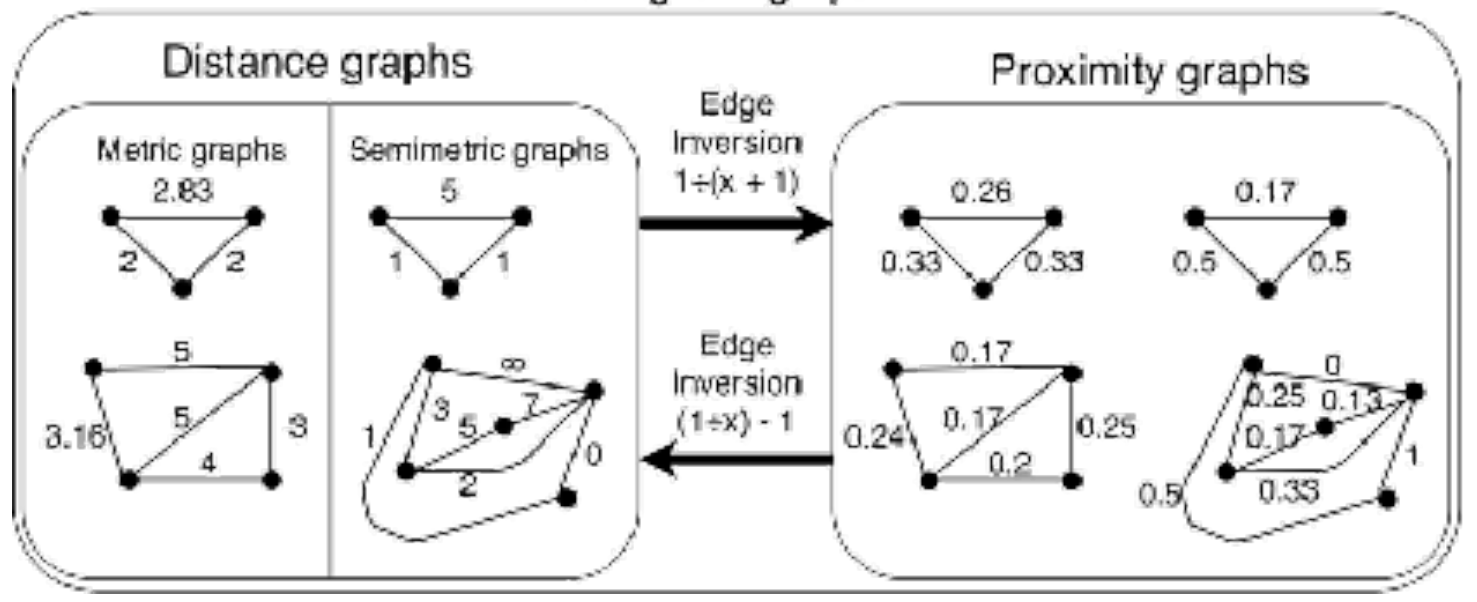

Figure 1: Illustration of different graph types and the terminology used to reference them in this article. See also Methods 2.2.

\section{Methods}

\subsection{Measurements and optimisation}

In this article, we define normative pathways and discuss a method for their detection, illustrating its performance on simulated data and in vivo images acquired in a case-control design. We present: (1) an index to measure the consistency of pathways between two nodes across a group of individuals - the Jaccard Edge Index; (2) an optimization problem that maximizes this index, thus identifying normative pathways, by analyzing the $k$ shortest pathways between two nodes; (3) an optimisation algorithm that heuristically estimates this problem, providing a practical means of finding normative pathways; (4) the behavior of the optimization algorithm and its ability to accurately identify normative pathways tested with simulated matrices where the ground truth is known; (5) a comparison of normative pathways to shortest pathways, in terms of edge composition, centrality measures, and efficiency, in a group of control adolescents; and (6) a derivation of a statistical method for the detection of differences between normative pathways in two groups of connectomes, and apply it to a case-control comparison between adolescents with a diagnosis of MDD and control individuals.

In common with the overwhelming body of prior work in functional connectomics, the Pearson correlation of time-series extracted from two nodes is the estimate of connectivity that weights the edges between the nodes in the connectome. However, we also applied the methods of identifying and comparing normative pathways when estimating connectivity with partial correlation (which regresses out the time series of every other node in its comparison), and normalized mutual information (which quantifies the shared information between two variables). We refer to different connectivity measurements as modalities, and in each experiment we compare across modalities.

\subsection{Terminology}


A graph, $G$ is defined as a set of nodes, or vertices $V$, connected by edges $E(G=\{V, E\})$, that may be directed or undirected, depending on whether edges have associated directionality. Functional connectomes are generally undirected graphs of which there are two types: weighted and unweighted (or binary) which, respectively, refer to graphs with and without numerical values associated with their edges. We use the term proximity graph when larger edge values represent stronger connections. Thus, in a proximity graph with edge values on [0,1], 0 represents a weak association and 1 represents a strong association. Conversely, we use the term distance graph when smaller edge values are associated with stronger (i.e. closer) connections and larger edge values are associated with weaker (i.e. more distant) connections. In distance graphs, edges may be metric or semi-metric depending on whether or not they satisfy the triangle inequality. Thus, an edge is semi-metric if it is not the shortest path between the two nodes it directly connects. See Figure 1 for a visual depiction of these different terms.

\subsection{Participants and MRI Data}

Control data were taken from a sample of 34 healthy adolescents ( 7 males and 27 females, aged 12 to 18 years, mean age $=15.7$, standard deviation $=1.45$ ) with no family history of depression, who were recruited by advertisement from local schools. Forty (40) were initially recruited, with a total of 6 excluded. All of the participants were rescanned six months later as part of a longitudinal study, with four excluded. Participants were processed using a 116-area anatomical connectome. For details on data acquisition and processing, see Appendices B and C.

\subsection{Deriving the semimetric connectome}

Due to controversies around interpretation of negative correlations between brain regions (Fox et al., 2009; Murphy et al., 2009), when constructing a graph from estimates of connectivity we first take the common step of setting the negative Pearson correlations to zero (Cao et al., 2014), and additionally set those correlations with an associated $p>0.05$ to zero (this value is, of course, arbitrary, but it is an effort to eliminate spurious connections). To convert the edges of this weighted proximity graph to a distance graph on which path finding algorithms may be applied, we use a mathematical construct called a t-norm that converts from $[0,1]$ to $[\infty, 0]$ using a version of the Dombi $t$-norm (Dombi, 1982; Simas, 2012):

$$
\begin{gathered}
f(x)=1 / x-1 \\
f(x)=\frac{1}{x}-1
\end{gathered}
$$

As these inverted weights may violate the triangle inequality, the distance graph is embedded in a semi-metric space in which path finding algorithms may be applied. To convert back from semi-metric distance space to a proximity space, we apply the inverse of Equation 1:

$$
\begin{gathered}
f^{-1}(x)=1 /(x+1) \\
f^{-1}(x)=\frac{1}{x+1}
\end{gathered}
$$

\subsection{Path length measurements}

Functional connectomes are most commonly represented as proximity rather than distance graphs, and thus it is convenient to also express paths in proximity space. We therefore find our path lengths by first converting the graph from a proximity to distance space (Equation 1), summing the distances, and then converting back from distance to proximity space (Equation 2).

Within a distance graph, the path length is the sum of the values of edges that make up a path between two nodes. Within the Pearson correlational space that has negative correlations set to 0 and using a Dombi $t$-norm to sum correlations, the path, $P$, from node $i$ to node $j$, consisting of correlations (i.e. edges) $\left\{P_{1}, P_{2}, \ldots P_{n}\right\}$ is summed to weight $W(P)$ : 


$$
\begin{gathered}
W(P)=1 /\left(\sum\{i=1\}^{\wedge}\{n\}\left(1 / P_{i}-1\right)+1\right) \\
W(P)=\frac{1}{\sum_{i=1}^{n}\left(\frac{1}{P_{i}}-1\right)+1}
\end{gathered}
$$

This is simply Equation 1 (the Dombi $t$-norm) embedded in a summation within Equation 2 (the inverse of the Dombi $t$-norm). See also Appendix D.

\subsection{Jaccard Edge Index}

When assessing the shortest pathways connecting nodes $i$ and $j$ in two functional connectomes, both may have similar lengths yet be routed through different brain regions. Thus, when comparing pathways connecting two areas across individuals, not only is the length $(W(P)$, Equation 3$)$ of the paths important, but also their edgewise composition. We perform this comparison by viewing a path as a set of edges.

The Jaccard index is a value between 0 and 1 that compares the composition of two sets:

$$
\begin{array}{r}
J(A, B)=|A \cap B| /|A \cup B| \\
J(A, B)=\frac{|A \cap B|}{|A \cup B|}
\end{array}
$$

If $J(A, B)=1$, then $A$ and $B$ are identical sets, and if $J(A, B)=0$, then $A$ and $B$ have no elements in common. Thus, taking the Jaccard Index of the edges of two paths gives a measure of their similarity; i.e., the number edges the paths have in common divided by the number of unique edges that compose the two paths. Across multiple paths, the index is averaged between each pairing of pathways. For example, suppose $\eta(G, i, j)$ returns the shortest path from nodes $i$ to $j$ for graph $G$. Then, for $N$ graphs, $\left[G^{1}, G^{2}, \ldots G^{N}\right]$, using Equation $3(W(P))$ to evaluate path lengths, this gives us an array $J$ with elements: 


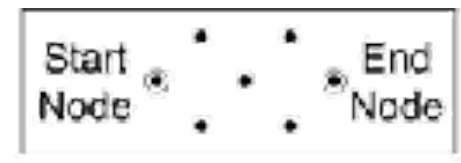

= shortest path

$=$ 2nd shortest path

E = Brd shortest path

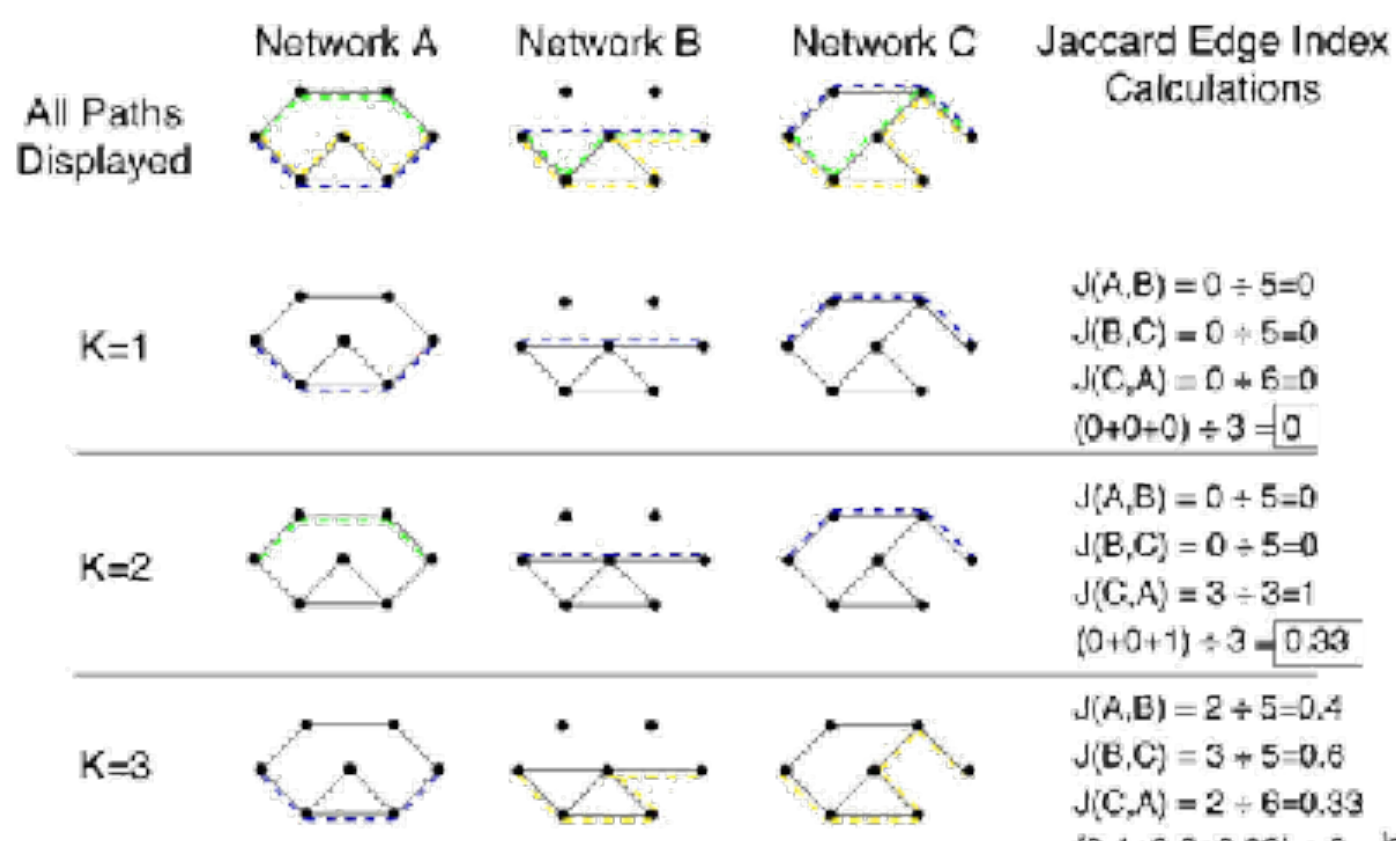

$\{0.4+0.6+0.33\} \div 3=0.44$

Figure 2: Optimal Jaccard Edge Index that is obtained when $K=\{1,2,3\}$ (i.e. when the first, second, and third shortest paths are considered) on a set of toy binary graphs. The top row displays, on the colored dotted lines, the three shortest paths of each of the three binary networks between the start and end nodes. The following three rows show which path would be selected in each of the three networks to obtain the optimal Jaccard Edge Index (if $K=2$, the two shortest paths are considered but not the third). The function $J(x, y)$ is the Jaccard Edge index for the paths considered between two given networks. Note that the first and second shortest paths for network $A$ are equal in length and the choice is arbitrary.

$$
\begin{gathered}
\mathrm{J}_{\mathrm{ij}}=2 /(\mathrm{N}(\mathrm{N}-1)) \times \sum\{\mathrm{x}=1\}^{\wedge}\{\mathrm{N}\} \sum\{\mathrm{y}=\mathrm{x}+1\}^{\wedge}\{\mathrm{N}\}\left(\left|\eta\left(\mathrm{G}^{\mathrm{x}}, \mathrm{i}, \mathrm{j}\right) \cap \eta(\mathrm{Gy}, \mathrm{i}, \mathrm{j})\right|\right) /\left(\left|\eta\left(\mathrm{G}^{\mathrm{x}} \mathrm{i}, \mathrm{j}\right) \cup \mathrm{n}(\mathrm{Gy}, \mathrm{i}, \mathrm{j})\right|\right) \\
J_{i j}=\frac{2}{N(N-1)} \sum_{x=1}^{N} \sum_{g=x+1}^{N} \frac{\mid \eta\left(G^{x}, i, j\left|\cap \eta\left(G^{y}, i, j\right)\right|\right.}{\mid \eta\left(G^{x}, i, j\left|\cup n\left(G^{y}, i, j\right)\right|\right.}
\end{gathered}
$$

See Figure 2 for an illustration of the Jaccard Edge Index on toy graphs.

The Jaccard Edge Index provides a measure of consistency of the shortest paths across a group of functional connectomes. If $J_{i j}=1$, then the same pathway connects nodes $i$ and $j$ in all connectomes; if $J_{i j}=0$, then the pathways connecting $i$ and $j$ do not have a common edge.

To find a global measurement of shortest path consistency, we took the average of the $n \times n$ matrix $J$, excluding redundant paths:

$$
\begin{aligned}
& J_{\text {global }}=2 /(n(n-1)) \sum\{i=1\}^{\wedge}\{n\} \sum\{i=j+1\}^{\wedge}\{n\} J_{i j} \\
& J_{\text {giane }}=\frac{2}{n(n-1)} \sum_{i=1}^{n} \sum_{i=j+1}^{2} d_{t}
\end{aligned}
$$


We refer to Equation 5 as the Jaccard Edge Index of the path connecting nodes $i$ and $j$, and to Equation 6 (the average of all Jaccard Edge Indices) as the Global Jaccard Edge Index.

\subsection{Normative pathways}

For a variety of reasons, the shortest paths may not be utilized in real-world graphs. To accommodate this perspective, we found the paths across a particular group that minimized path length whilst maximizing edge sharing. We refer to these pathways as normative.

We define the normative pathways as the selection of pathways that maximise the Jaccard Edge Index over a selection of the $k$ shortest pathways, across a group of connectomes. Informally, this means that, instead of identifying the optimally shortest pathways connecting two nodes (which may differ depending on the connectome), we identify a set of slightly suboptimal pathways that pass through similar areas.

Thus, to identify the normative pathways between two nodes across individuals, we search for the $k$ shortest paths that maximize $J$. To do this, we use Yen's K-Shortest Path algorithmlcitep\{Yen1971\}, which finds the $k$ shortest pathways by searching around each edge in the shortest path (found by Dijkstra's algorithm) and ranking the resulting paths.

Suppose that $k(G, i, j)$ returns the $k$ th shortest path for a given connection from nodes $i$ to $j$ in graph $G$, searching across a maximum of $\mathrm{K}$ paths, for computational feasibility. Suppose, also, that $k_{l}$ is the $k$ th path selected for network I (so that, for instance, $\left[k_{1}, k_{2}, k_{3} \ldots k_{N}\right]=[1,18,2 \ldots 9]$ ). For a group of $N$ graphs, $\left[G^{1}\right.$, $\left.G^{2} \ldots G^{N}\right]$, the normative pathways yield the maximum $J_{i j}$ in the following equation:

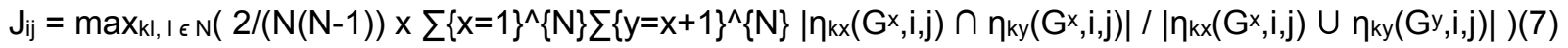

$$
\begin{aligned}
& J_{i j}=\max _{k_{i}, i \in N}\left(\frac{2}{N(N-1)} \sum_{x=1}^{N} \sum_{y=x+1}^{N} \frac{\left|m_{k_{-}}\left(G^{x}, i, j\right) \cap r_{i k_{\mathrm{s}}}\left(G^{x}, i, j\right)\right|}{\left.\left|m_{k_{x}}\right| G^{*}, i, j\right) \cup r_{i k_{\mathrm{s}}}\left(G^{s}, i, j\right) \mid}\right)
\end{aligned}
$$

An instance of the maximum Jaccard Edge Index found for $K=\{1,2,3\}$ on a set of toy graphs can be seen in Figure 2.

When $K=1$ in the Jaccard Edge Index Maximization problem, this simply returns the shortest paths. As $K$ increases, pathways become more consistent across individuals (i.e., the Jaccard Edge Index increases), although the path lengths become longer.

\subsection{Identification of normative pathways by Maximization of the Jaccard Edge Index}

The maximisation problem expressed in Equation 7 is nontrivial to solve, and must be estimated via a heuristic. For each of $N$ graphs, the $k$ shortest paths are computed (a total of $N x k$ paths). With each graph contributing one path, the set of $N$ pathways is found that share the most common edges, thus maximizing the Jaccard Edge Index, $J_{i j}$.

We can maximise each $J_{i j}$ value independently. Given $N$ connectomes and starting from $\forall l \in N, k=1$, we may iterate through $I \in N$ in random order, finding the value $k_{l} \in K$ that maximizes $J_{i j}$. We cease when no further increases in $J_{i j}$ or can be made for $\exists / \in N: k_{l}<K$. We refer to this algorithm as the Jaccard Edge Index Maximization Algorithm.

Informally, iterating through the set of functional connectomes in random order, we test which of the $k$ shortest paths connecting nodes $i$ and $j$ in a particular graph is most similar to the current set of paths from all other connectomes (Equation 7). We then use the path that maximizes the Jaccard Edge Index, stopping when no further increments can be made to the Index.

We tested the algorithm on all node pairings of a test group of 34 control participants across $K=[1 \ldots 20]$, testing its ability to raise the Jaccard Edge Index (and decrease the average path length) as $K$ increases.

\subsection{Ground-Truth Simulation of Randomized Matrices}


To test the efficacy of this algorithm in identifying normative pathways, we simulated randomized matrices with seeded ground-truth pathways and applied the Jaccard Edge Index Maximization Algorithm. We did so first by sampling time series from MDD and control participants, combining the two datasets to increase the overall size of our sampling pool. For each test, we then averaged 3 to 7 nodes (varying path length from 2 to 6 ) with another timeseries, randomly selected from another participant. In half of our tests, the same random variable was used for all nodes in a particular instance (effectively seeding a subgraph), while the other half saw an independent variable seeded in each node pairing. We then measured the Pearson's correlation, partial correlation, and normalized mutual information matrices for each test.

When seeding the path, we varied path length from 2 to 6 and signal-to-noise ratio of the simulated effects from 0 to 2 with increments of 0.025 , and then measured the percentage of times in 20 tests that the seeded pathway was present in the 20 shortest pathways connecting the respective nodes, for a total of 19,200 simulations. Following this, we tested whether the Jaccard Edge Index Maximization Algorithm converged on the simulated pathway between the two seeded nodes.

\subsection{Comparing Edge Usage of Normative Pathways to that of Shortest Pathways}

As a means of displaying which edges are more utilized between the shortest pathways and the normative pathways, we ran the Jaccard Edge Index Maximisation Algorithm on the test group of 34 control adolescents $(K=20)$, finding all normative pathways for each node pairing across all participants. We separately find the shortest pathways $(K=1)$. We then count, for each edge in each participant's connectome the number of times that a normative and a shortest pathway utilizes it, giving a groupwise aggregate. The counts of normative and shortest usage are each normalized to a z-score and subtracted from one another, giving each edge in the connectome a score that approximates its increased utilisation by either normative or shortest pathways. Informally, this shows us which areas and connections normative pathways tend to utilize more than shortest pathways.

\subsection{Closeness Centrality and Efficiency of Normative pathways}

To summarize normative pathways in a connectome, we analyzed modified versions of two common graph measurements: closeness centrality (Bavelas, 1950; Freeman, 1979) and average efficiency (Latora and Marchiori, 2001). In our context, the closeness centrality of node $i$ is the average path length $(W(P)$, Equation 3$)$ of the normative paths extending from node $i$ to all other nodes in that graph. Average efficiency is the average of all closeness centralities for a particular graph.

Both measures were modified to consider the normative $(K=[2 \ldots 20])$ pathways, rather than only the shortest $(K=1)$ pathways.

Given a set of paths from $i$ to all other $n$ nodes, $\left\{P_{i, 1}, P_{i, 2 \ldots}, P_{i, i-1,1} P_{i, i+1} \ldots P_{i, n}\right\}$, we define closeness centrality for node $i$ as

$$
\begin{aligned}
& C_{i}=1 /(n-1) \times \sum\{j \in n, j \neq i\} W\left(P_{i, j}\right) \\
& C_{i}=\frac{1}{n-1} \sum_{j \in=, j \neq i} W\left(P_{i, j}\right)
\end{aligned}
$$

The variance of these centralities with increasing paths $(K=1,2, \ldots 20)$ was also recorded, as well as the derivative of this value with respect to the number of paths used, since we are interested in the stability of these measurements as the set of shortest paths increases in number.

Additionally, the average efficiency of the graphs as $K$ increased was calculated to observe the Jaccard Edge Index Maximization Algorithm's effect on global path length measurements.

$$
E=1 / n \times \sum\{i=1\}^{\wedge}\{n\} C_{i}
$$




$$
\bar{E}=\frac{1}{n} \sum_{i=1}^{n} C_{i}
$$

We calculated these values and variances for the test and retest control groups and plotted them against $K$. See Appendix E for information on the relationship between the Jaccard Edge Index and the shortest pathways on the average connectome.

\subsection{Cross-group normative pathway comparison}

To apply these concepts to case-control studies, we look at a statistical method of comparing the normative pathways in two separate groups of connectomes, in order to detect the areas in which normative pathways converge in one group but not another and vice-versa. This method focuses on finding differences in the Jaccard Edge Indices of normative pathways between groups that show statistically significant differences, with significance found via comparison to a null model.

For two groups, $A$ and $B$, the $n x n$ Jaccard Edge Indices, $J_{A}$ and $J_{B}$ respectively, are obtained for each separately:

$$
\begin{gathered}
J_{\text {diff }}=J_{A}-J_{B} \\
J_{d i f f}=J_{A}-J_{D}
\end{gathered}
$$

High values of elements in the resulting matrix, $J_{\text {diff, }}$ are node pairings with normative pathways that converge to a greater in extent in Group A than Group B, while low values are node pairings with normative pathways with greater convergence in Group B, but not Group A.

To find the statistically significant values of $J_{\text {diff, }}$ we created n null model matrices, $\left[J_{N 1}, J_{N 2} \ldots, J_{N 3}\right]$, each found by applying the Jaccard Edge Index Maximization Algorithm samples of connectomes randomly assigned to each of the two groups preserving the group sizes of the observed sample. For each possible pairing, Jdiff was calculated (Equation 10) to give a total of $n x(n-1)$ different $J_{\text {diff }}$ matrices. Subsequently, the distribution of $n x(n-1)$ values under the null hypothesis was derived for each connection between nodes $i$ and $j$. By taking the mean and standard deviation of these distributions, we converted the values of the observed Jdiff matrix into a z-score for each node pairing:

$$
\begin{aligned}
\mathrm{J}_{\mathrm{Z}}=\left(\mathrm{J}_{\text {diff }}-\left\langle\mathrm{J}_{\mathrm{Ni}} \forall \mathrm{i} \epsilon \mathrm{n}\right\rangle\right) / \sqrt{ }\left(\left\langle\mathrm{J}_{\mathrm{Ni}}{ }^{2} \forall \mathrm{i} \epsilon \mathrm{n}\right\rangle-\left\langle\mathrm{J}_{\mathrm{Ni}} \forall \mathrm{i} \epsilon \mathrm{n}\right\rangle^{2}\right) \\
J_{z}=\frac{J_{\text {diff }}-\left\langle J_{N_{i}} \forall i \in n\right\rangle}{\sqrt{\left\{J_{N_{i}}{ }^{2} \nabla i \in n\right\rangle-\left\langle J_{N_{i}} \nabla i \in n\right\rangle^{2}}}
\end{aligned}
$$

The $z$-scores were converted to $p$-values using the Fisher $Z$ transformation. Correction for multiple comparisons was undertaken using false discovery rate (Benjamini and Hochberg, 1995) and thresholding at $q=0.05$, identifying the elements of $J_{\text {diff }}$ that were statistically significant between groups.

We performed this analysis on the MDD and control groups, which gives a number of normative pathways for each group. We quantified the number of times each edge was used across participants for each group, then determined to which regions of the brain these edges connected and which they passed through most often, using the same normalisation technique as above. As a means of validation, we compared these results with different studies including meta-analyses performed in MDD-control connectivity, primarily with adult participants. We counted the number of edges composing normative pathways that were significantly different that crossed through each area. The areas were then ranked and compared with those areas found to be functionally different between MDD and control adult groups in the meta-analysis of Kaiser et al. (2015). When a different parcellation, or no parcellation, was used, 
we manually found the closest corresponding area of the brain in the Automated Anatomical Labelling (AAL) parcellation (Tzourio-Mazoyer et al., 2002).

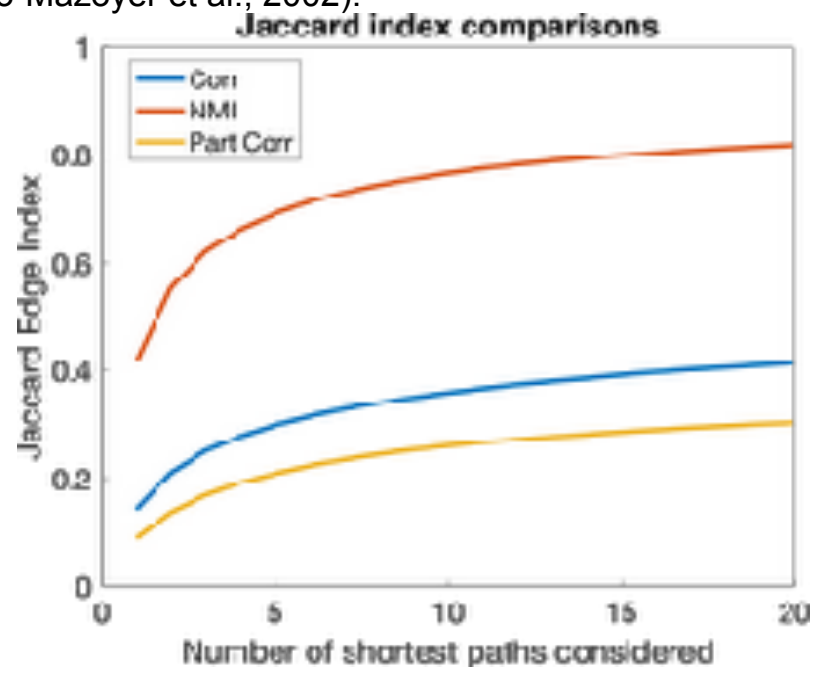

Figure 3: Comparison of the Jaccard Edge Indices with normalized mutual information, partial correlation, and correlation modalities in the test group of control participants. This displays the levels of consistency in pathways for each of the modalities. As we can see, Normalized Mutual Information offers the highest path consistency overall, being 0.80 at $K=20$.
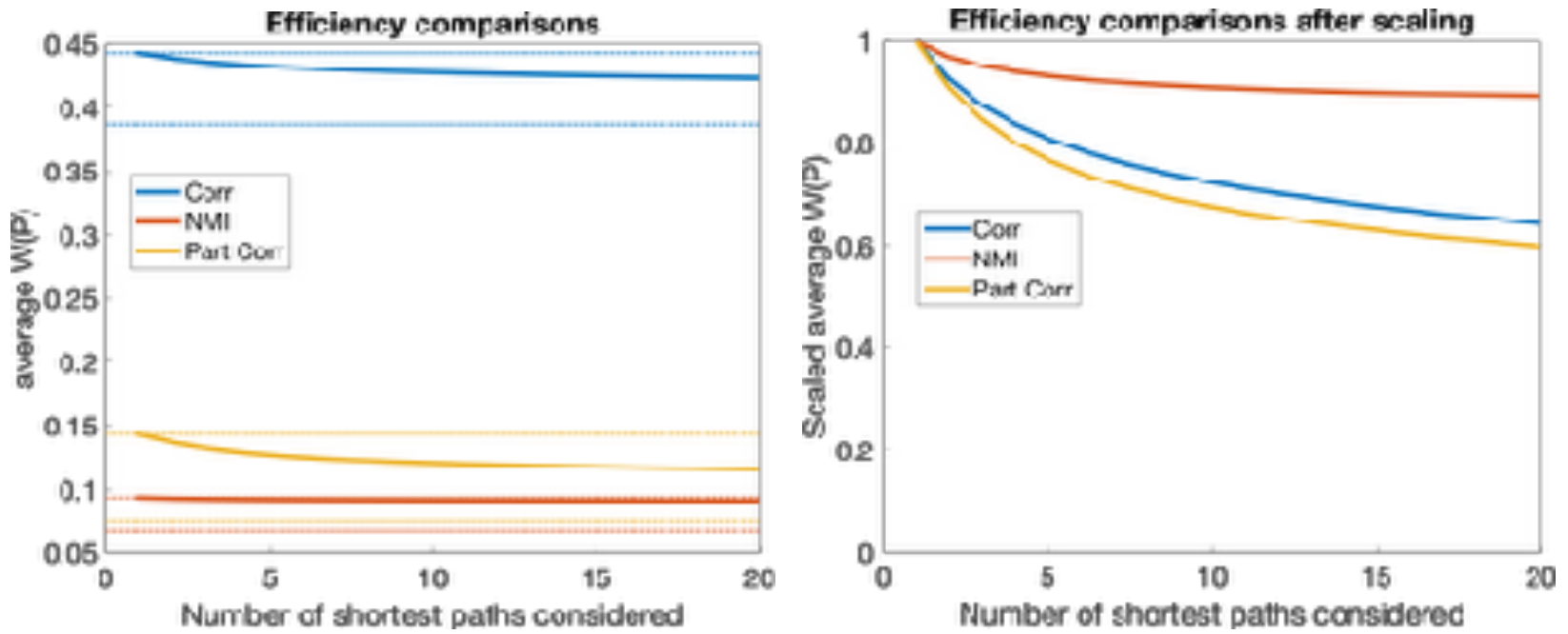

Figure 4: Comparison of the average path lengths (i.e. efficiency) for different modalities over the test group of control participants as $K$ increases. The left side shows the average efficiency of connectomes in the control group as more pathways are optimized for consistency; upper dotted lines represent the efficiency at $K=1$, while the lower dotted lines represent the efficiency of the mean graph (see Figure E.1), which offers a way of scaling these lines. The right shows these three lines after scaling. See Methods 2.11. 


\begin{tabular}{|l|l|l|l|l|l|l|l|l|l|l|l|}
\hline & & & & & & & \\
\hline
\end{tabular}

Table 1: Mean pathway recovery percentages across all tests and signal-to-noise ratios for randomized simulations. See Figure 7.

\begin{tabular}{|l|l|l|l|l|l|l|l|l|l|l|}
\hline & & & & & & & & \\
\hline
\end{tabular}

Table 2: Mean Jaccard Edge Indices across all signal-to-noise ratios for randomized simulations. See Figure 8.

\section{Results}

\subsection{Performance of the Jaccard Edge Index Maximization Algorithm}

Figure 3 shows the improvements in the Jaccard Edge Index as $K=[1 \ldots 20]$ increases, while Figure 4 shows the decrease in overall efficiency (indicating path length) as $K=[1 \ldots 20]$ increases. The matrices for $K=[0,10,20]$ are shown in Figure 5 . Each modality saw a sharp increase in internal consistency of its pathways by the application of the Jaccard Edge Index Maximization Algorithm, utilizing a greater distribution of edges in composition of paths (Figure 6), with a small loss in overall efficiency of these paths. The most consistent pathways were seen with connectivity estimated by normalized mutual information at $K=20$, with $J_{\text {global }}=0.80$; in other words, the normative pathways of connectomes using the normalized mutual information modality, on the whole shared the fewest edges, but exhibited the most internal consistency.

\subsection{Ground-truth simulation with randomized matrices}

We simulated randomized matrices that maintained a small-world structure and degree distribution of functional connectomes by randomly sampling time series from the control and MDD datasets. The results are shown in Figure 7. We then applied the Jaccard Edge Index Maximization Algorithm to each set of 20 matrices having 
the same seeded path, signal-to-noise ratio, and edge independence. The means of each of the recovery percentages (the percent of tests in which the seeded path appeared in the 20 shortest paths) and the Jaccard Edge Index, across all tests, path lengths, and signal-to-noise ratios, are shown in Tables 1 and 2, respectively.

When independent variables were seeded for each edge, partial correlation saw the highest success in recovering the seeded pathway, uncovering paths an average of $59.94 \%$ of the time on paths of length 6 , while the highest average recovery rate for correlation and normalized mutual information for paths of length four and above was $5.49 \%$ (see Table 3 ). When a single, global variable was seeded for each path, however, partial correlation did a poorer job of recovering these pathways (as one may expect, since the single random variable, appearing in multiple time series, is regressed out), having a $5.6 \%$ average recovery rate for paths of length 4 and $0 \%$ for lengths 5 and 6 . Correlation and normalized mutual information modalities had a $44.94 \%$ and $41.30 \%$ average recovery rate, respectively, for paths of length 4.

In general, the Jaccard Edge Index converged in the presence of a normative path with a high signal-to-noise ratio, regardless of path length. This indifference to path length is likely due to the convergence of the algorithm on another path that utilized individual edges of the seeded path. See Table 2 and Figure 8.

Due to the recovery percentages, this result indicates that the Jaccard Edge Index Maximization Algorithm is capable of finding seeded pathways in data, although this is dependent on both the modality and the exact method of seeding the pathways (i.e., whether we use one random variable per edge or different ones). Although this is an imperfect analogy for real-world fMRI data, it does offer an idea of the baseline efficacy of the algorithm.

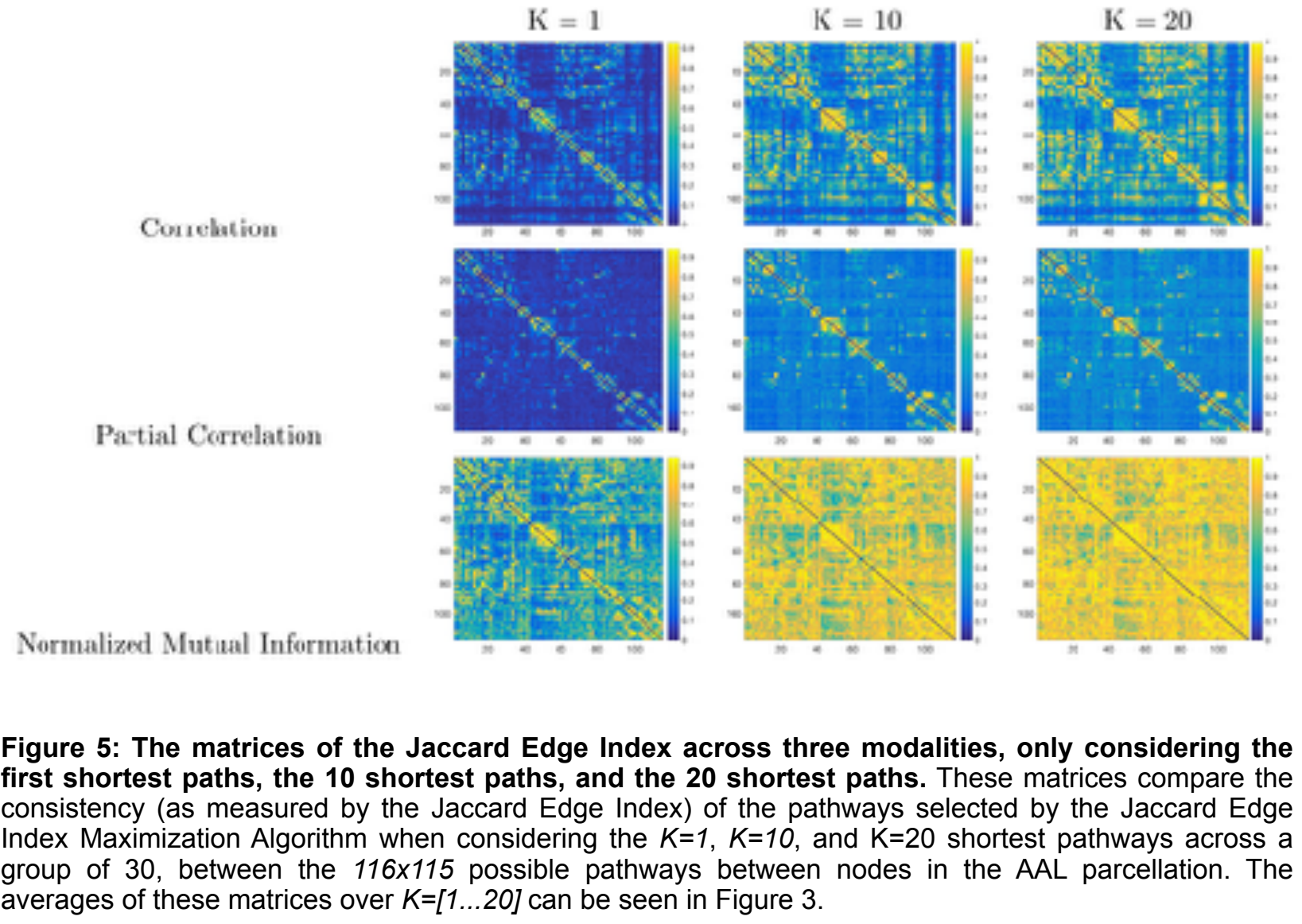




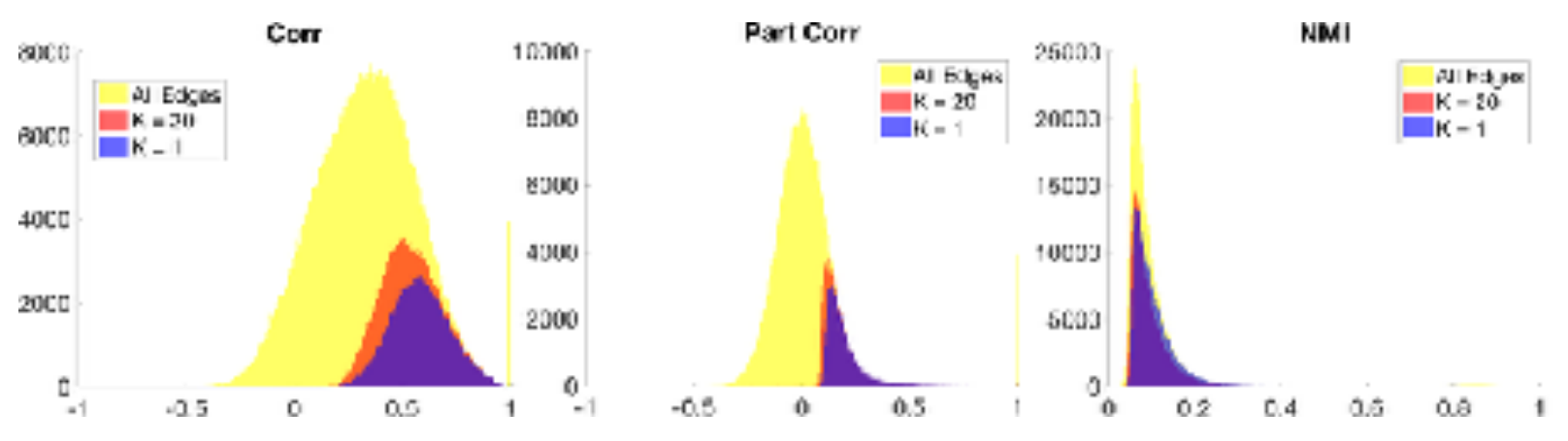

Figure 6: Comparison of overall edge utilization between the shortest pathways $(K=1)$ and the normative pathways $(K=20)$ for the 34 control participants. In aggregate, the normative pathways utilized a greater proportion of the edges than the shortest pathways. Note that, for Pearson's correlation and, to a greater degree, partial correlation, the composition of pathways is strongly affected by the thresholding of the negative edges.

\subsection{Comparing Edge Usage of Normative Pathways to that of Shortest Pathways}

Figure 9 shows which edges and nodes were utilized more, in aggregate, by normative pathways $(K=20)$ than shortest pathways $(K=1)$, between modalities. As expected, normative pathways utilized a wider range of edges, including weaker ones. Average path lengths of the normative pathways for Pearson's correlation, partial correlation, and normalized mutual information modalities were 3.48, 3.93, and 2.12, respectively, compared to $3.39,2.37$, and 3.35 for the shortest pathways.

Normative pathways were more frequently routed through nodes along the upper cerebellum and the border between the brain hemispheres, where one would expect anatomical pathways to bottleneck. This is most apparent with connectomes constructed with partial correlations which showed particular increased traversing of pathways through the striatum, which receives projections from the entire cerebral cortex. Connectomes constructed with normalized mutual information showed large increases in the left and right middle cinguli; anatomically, the cingulum is a highly connected area (Hagmann et al., 2008) that acts as a global connector for other functional networks (Guimera et al., 2007; Leech and Sharp, 2014). Finally, connectomes constructed with Pearson's correlation showed large increases in parts of the upper cerebellum and vermis, and along areas directly bridging the two hemispheres; the most apparent exception, however, is between the two superior temporal lobes.

These differences suggest that normative pathways vary depending on the modality. Considering the differing values of individual edges in each connectome and the edge utilisation in the $K=1$ shortest pathways and the $K=20$ normative pathways (Figure 3 ), this is more than likely due to inherent differences in the modalities. 

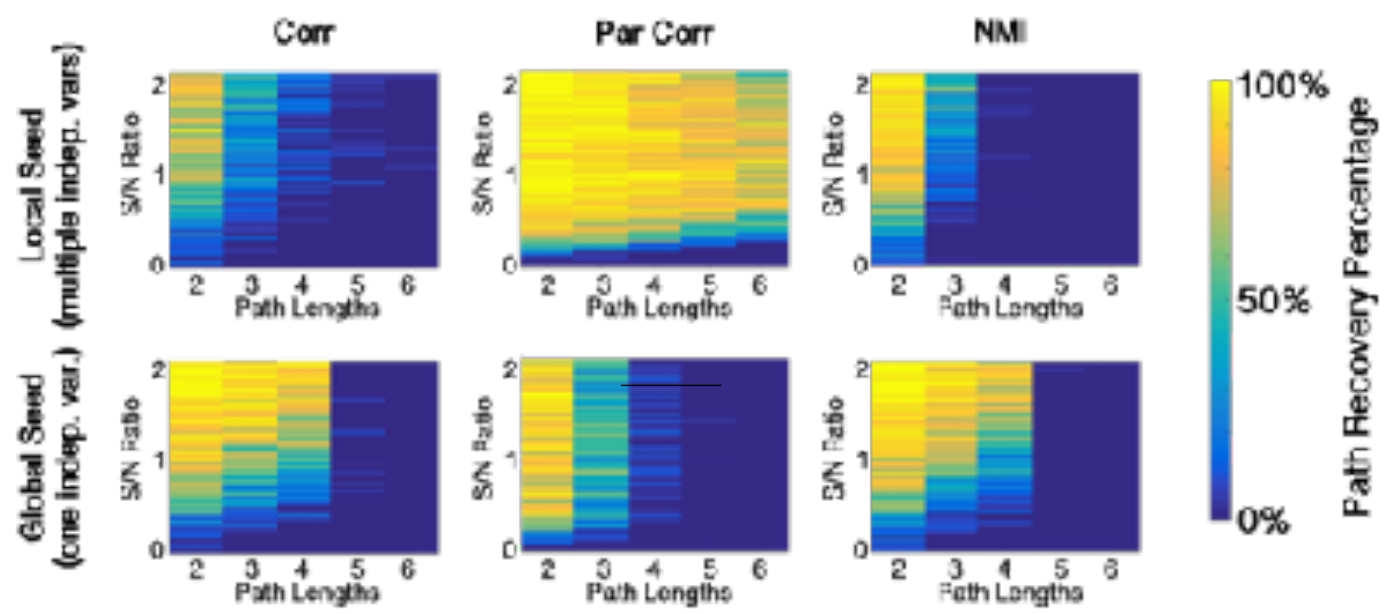

Figure 7: Percentage of times that seeded pathways appeared in top 20 shortest pathways in simulated matrices. In the top row ("Local"), one random variable was used for each edge in the path; in the bottom row ("Global"), one random variable was used for the entire path, effectively seeding a subgraph into the time series. See Section 2.9.
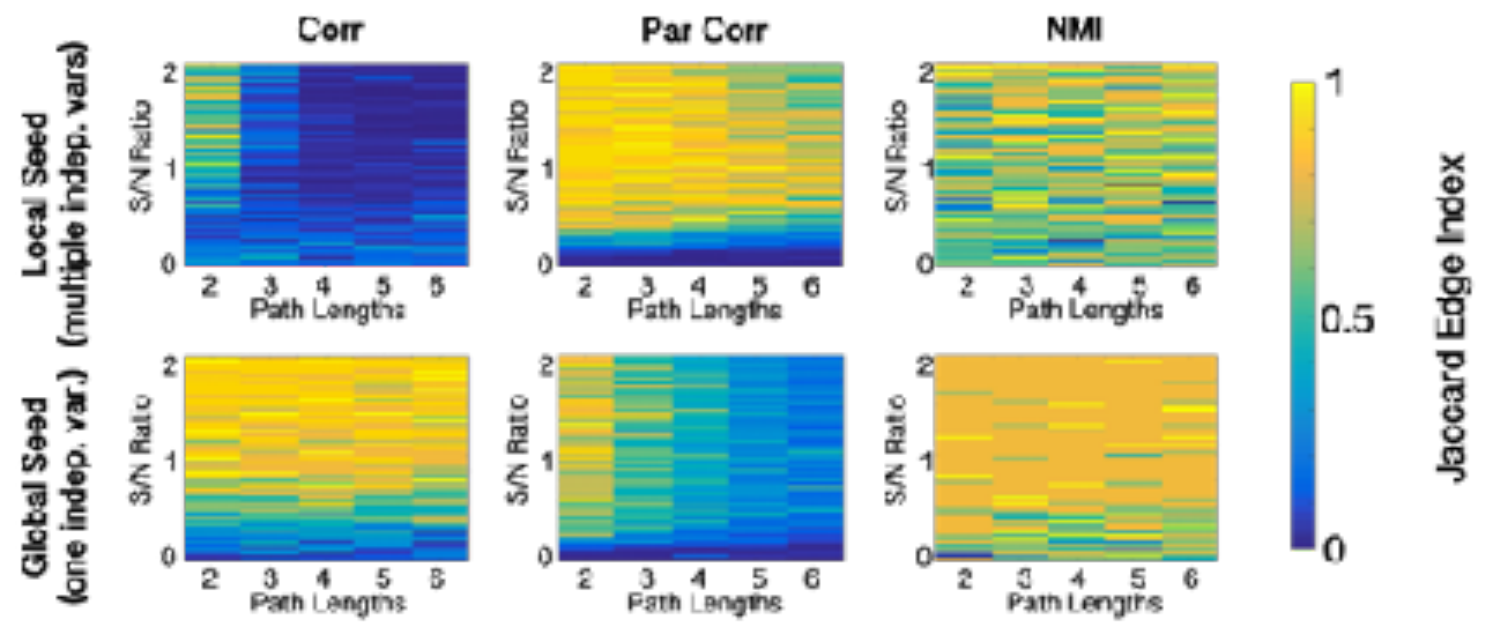

Figure 8: The Jaccard Edge Indices from the simulations in Figure 7. These show that, in the presence of real paths, the Jaccard Edge Index converges, even if it does not necessarily converge on the exact path that was seeded.

\subsection{Closeness Centrality and Efficiency of Normative pathways}

We measured the closeness centrality and the efficiency of normative pathways in the test group of 34 control participants for $K=[1 \ldots 20]$. As noted above, the efficiency of normative pathways for all three modalities decreases as $K$ increases, indicating that closeness centralities, on average, decrease. This is trivially true. However, we also measured the variance of the closeness centralities across all brain areas in all participants as $K$ increased, finding that these variances, on average, increase as $K$ increased. The average variance of these centralities (displayed as the red lines in Figure 10) monotonically increased except in the case of correlation, which reached its minimum at $K=4$ and monotonically increased thereafter. While it may be thought that normative pathways provide more stability in their global measurements than shortest pathways, this appears to not be the case in the test group. However, in the retest group of 30 control participants (scanned six months later), the variance of all three modalities 
tested decreased monotonically as more paths were considered (Figure 11). Thus, correlation displays a consistent decrease in its closeness centrality variance with this algorithm for up to $K=4$, though this is not replicated for partial correlation and normalized mutual information

While we can conclude that the algorithm is effective in recovering normative pathways on real-world data, with substantial differences between modalities, the question of whether this algorithm has an effect on the consistency of measurements of these pathways within a group is inconclusive.

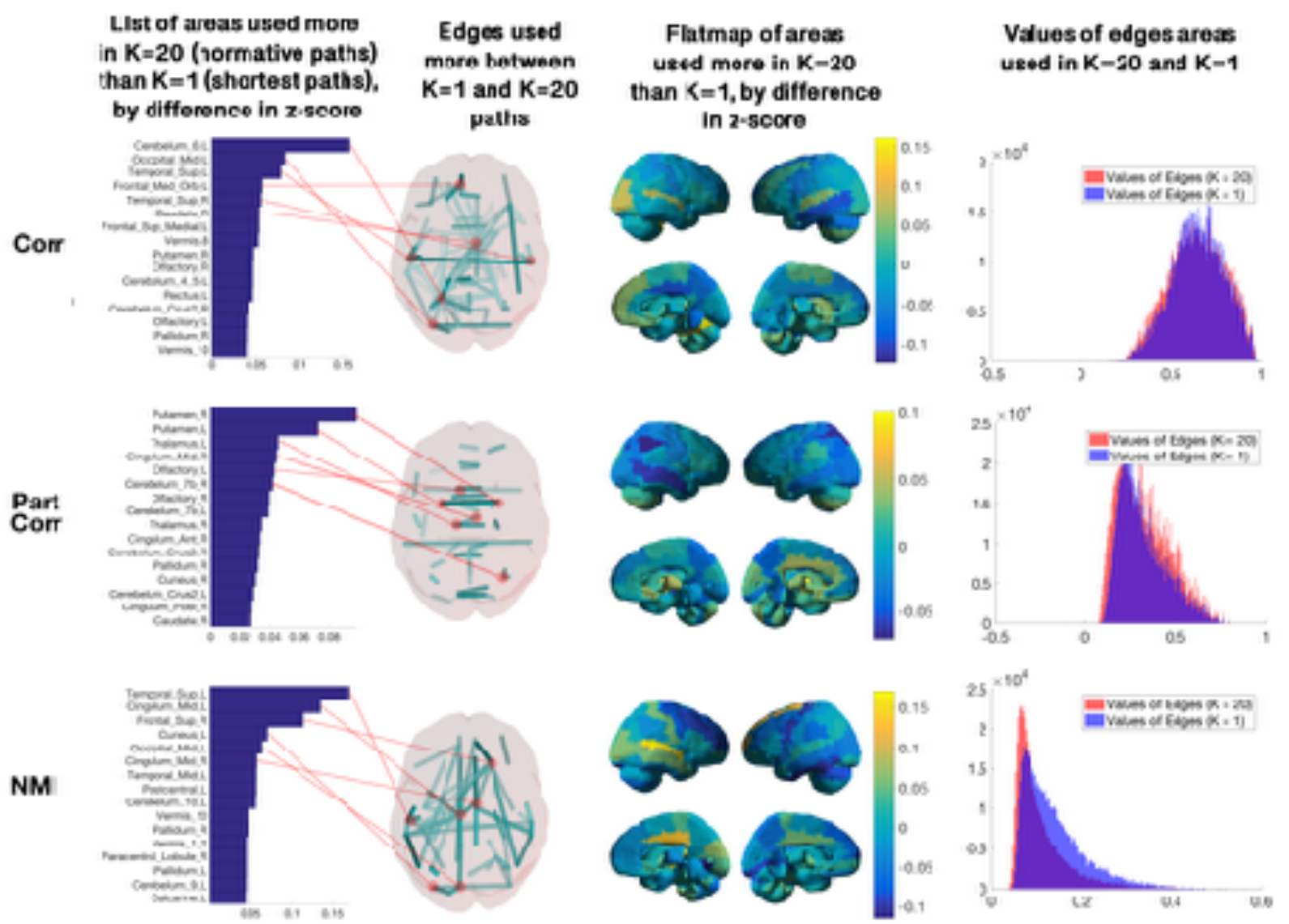

Figure 9: A display of which edges are more utilized between the shortest pathways and the normative pathways in the control participants. This visualization shows the difference in edge usage between $K=1$ in the Jaccard Edge Index Maximization Algorithm (i.e., when only the shortest paths are considered) and when $K=20$ (i.e., when the most consistently occurring, normative pathways are used). We counted the number of each times an edge appeared in a pathway when $K=1$ and $K=20$ across the 34 control participants, normalized these values to have the same mean and variance, and subtracted these normalized counts in $K=1$ from those in $K=20$, giving each edge a difference in $z$-score; the results are visualized in the second column, while the first column shows the hubs in the parcellation whose outgoing edges showed the greatest increments in utilization between $K=1$ and $K=20$; the flatmaps in the third column shows this across the whole brain. 

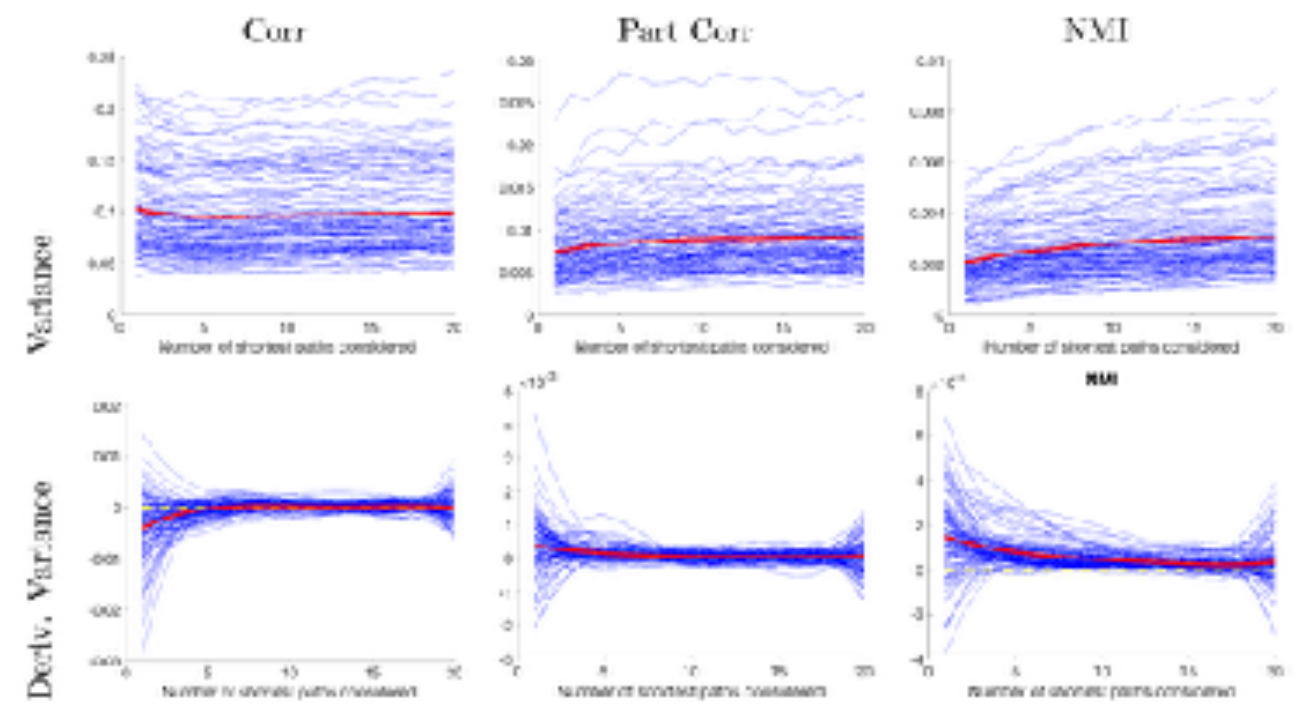

Figure 10: Variance of closeness centrality for all 116 nodes in the control group. (Above) The variance of the closeness centrality of each node, depending on the modality analyzed. Each blue line indicates the closeness centrality of one particular node in the parcellation, while the red line is the average. In general, higher variance is associated with a higher centrality value. (Below) The derivatives of these variances over path lengths considered after being fitted to a polynomial curve, which makes their fluctuations more evident. Note that, in these graphs, the order of magnitude is different, and these are meant to compare merely the fluctuations in variance per modality as the number of paths considered increases. Also note that the red lines are not true sums of variances in the true statistical sense, but are mainly used for display purposes to show general trends.

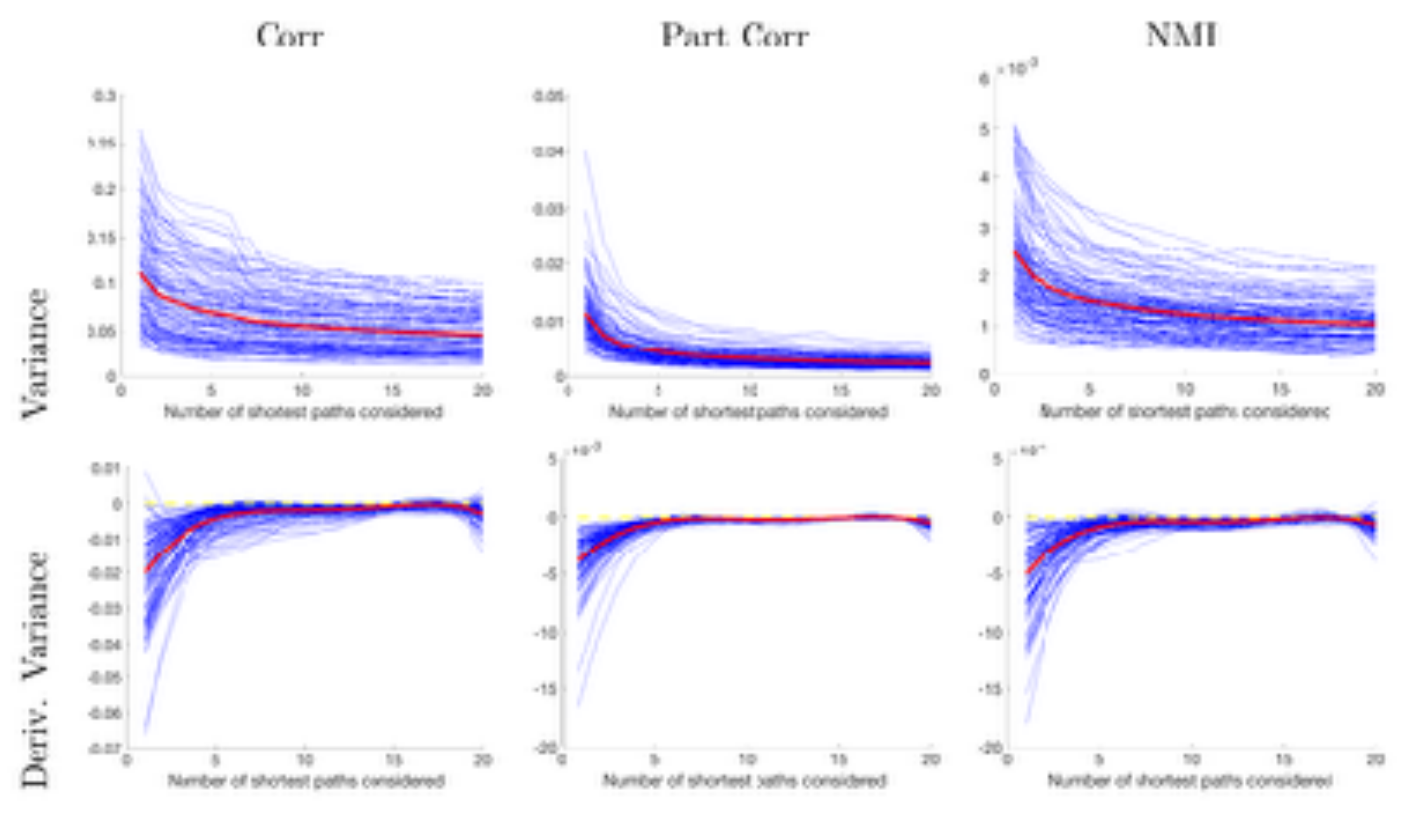

Figure 11: The same closeness centrality variance results as Figure 10 on the retest group. The retest group showed decreasing variances on its closeness centrality values, whereas the original group only showed this behavior in correlation. 


\begin{tabular}{|c|c|c|c|c|c|}
\hline \multicolumn{2}{|c|}{ Ventral Attention } & Frontoparietal & Default Mode & Affective & \\
\hline Corr & & $\begin{array}{l}\text { Controls } \\
\text { Par. Corr }\end{array}$ & \multicolumn{2}{|r|}{$\mathrm{NMI}$} & \\
\hline Anat. Area & \# Edges & Anat. Area & \# Edges & Anat. Area & \# Edges \\
\hline Cerebelum $6 \mathrm{~L}$ & 1186 & Postcentral L & 362 & SupraMarginal R & 70 \\
\hline Occipital Sup L & 626 & Precentral L & 208 & Occipital Sup L & 68 \\
\hline Cerebelum Crus1 L & 624 & Occipital Sup R & 176 & Cerebelum $10 \mathrm{R}$ & 34 \\
\hline Occipital Mid L & 538 & Cuneus $\mathrm{R}$ & 172 & Pallidum L & 34 \\
\hline Cerebelum Crus2 R & 446 & Calcarine L & 142 & Parietal Sup L & 34 \\
\hline Frontal Mid R & 400 & Vermis 12 & 132 & Calcarine $\mathrm{R}$ & 34 \\
\hline Temporal Sup L & 348 & Frontal Sup Medial R & 132 & Rectus L & 34 \\
\hline Caudate R & 348 & Rectus R & 130 & Frontal Med Orb R & 34 \\
\hline Vermis 6 & 312 & Vermis 9 & 122 & Frontal Sup Medial L & 34 \\
\hline Frontal Sup Medial L & 302 & Rectus L & 118 & Olfactory $\mathrm{R}$ & 34 \\
\hline Cuneus L & 294 & Occipital Mid R & 108 & Rolandic Oper R & 34 \\
\hline Temporal Sup R & 272 & Cuneus L & 106 & Frontal Inf Oper R & 34 \\
\hline Cingulum Mid L & 264 & Olfactory $\mathrm{R}$ & 106 & Frontal Sup Medial R & 8 \\
\hline Frontal Sup R & 256 & Cerebelum Crus2 L & 100 & Cerebelum 6 R & 2 \\
\hline Supp Motor Area R & 254 & Fusiform R & 94 & Temporal Sup R & 2 \\
\hline Precuneus L & 244 & Calcarine $\mathrm{R}$ & 86 & Precuneus L & 2 \\
\hline Frontal Inf Oper R & 234 & Lingual L & 76 & SupraMarginal L & 2 \\
\hline Cerebelum Crus1 R & 220 & Paracentral Lobule L & 74 & Lingual L & 2 \\
\hline Cingulum Mid R & 192 & Olfactory L & 74 & Cingulum Post $\mathrm{R}$ & 1 \\
\hline Frontal Sup Medial R & 192 & Frontal Sup Medial L & 72 & Frontal Mid R & 2 \\
\hline Cerebelum $6 \mathrm{R}$ & 188 & Rolandic Oper L & 72 & - & - \\
\hline Corr & & $\begin{array}{c}\text { MDD } \\
\text { Par. Corr }\end{array}$ & & $\mathrm{NMI}$ & \\
\hline Anat. Area & \# Edges & Anat. Area & \# Edges & Anat. Area & \# Edges \\
\hline Insula R & 384 & Temporal Mid R & 672 & Supp Motor Area R & 792 \\
\hline Temporal Pole Sup R & 268 & Temporal Sup R & 487 & Precentral L & 702 \\
\hline Fusiform L & 260 & Temporal Mid L & 296 & Postcentral L & 698 \\
\hline ParaHippocampal R & 258 & Frontal Sup Medial L & 272 & Cingulum Mid L & 634 \\
\hline Frontal Mid R & 240 & Temporal Inf R & 236 & Temporal Pole Sup R & 588 \\
\hline Fusiform R & 178 & Frontal Sup Medial R & 228 & Frontal Mid R & 564 \\
\hline ParaHippocampal L & 174 & Frontal Mid R & 202 & Cerebelum $45 \mathrm{~L}$ & 560 \\
\hline Lingual L & 172 & Cingulum Mid L & 188 & Rolandic Oper L & 544 \\
\hline Temporal Inf L & 164 & Cerebelum Crus1 R & 178 & Cerebelum $6 \mathrm{~L}$ & 524 \\
\hline Insula L & 164 & Frontal Sup R & 172 & Precentral R & 524 \\
\hline Frontal Inf Orb R & 164 & Cerebelum Crus2 R & 170 & Fusiform L & 514 \\
\hline Putamen L & 150 & Frontal Mid L & 162 & Frontal Med Orb L & 494 \\
\hline Frontal Inf Tri R & 96 & Cerebelum Crus2 L & 155 & ParaHippocampal R & 484 \\
\hline Temporal Pole Sup L & 92 & Cerebelum Crus1 L & 150 & Frontal Sup L & 480 \\
\hline Cerebelum $6 \mathrm{R}$ & $\overline{90}$ & Calcarine L & 142 & Supp Motor Area L & 478 \\
\hline Cerebelum $45 \mathrm{R}$ & 86 & Frontal Sup L & 142 & Insula R & 470 \\
\hline Lingual $\mathrm{R}$ & 86 & Cingulum Mid R & 140 & Thalamus L & 468 \\
\hline Cingulum Mid $\mathrm{R}$ & 86 & Caudate $\mathrm{R}$ & 136 & Cerebelum $8 \mathrm{~L}$ & 444 \\
\hline Rolandic Oper R & 84 & Temporal Sup L & 132 & ParaHippocampal L & 432 \\
\hline Cerebelum $9 \mathrm{R}$ & 82 & Fusiform L & 126 & Cerebelum $6 \mathrm{R}$ & 426 \\
\hline Cerebelum Crus2 R & 82 & Calcarine $\mathrm{R}$ & 122 & Frontal Sup Orb R & 400 \\
\hline
\end{tabular}

Table 3: Areas with the most unique normative pathways. Shown is the 20 areas through which the most normative pathways unique to that group (see Figure 12) pass through. Highlighted are those anatomical areas in which differences in connectivity between adult with MDD and control participants. 
Controk

Corr
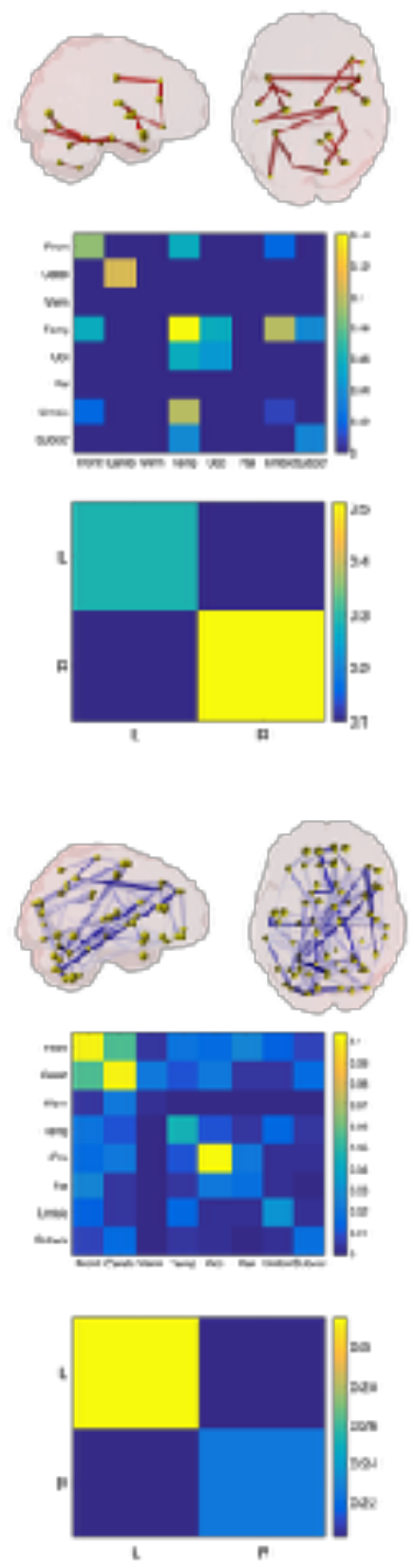

Par Corr
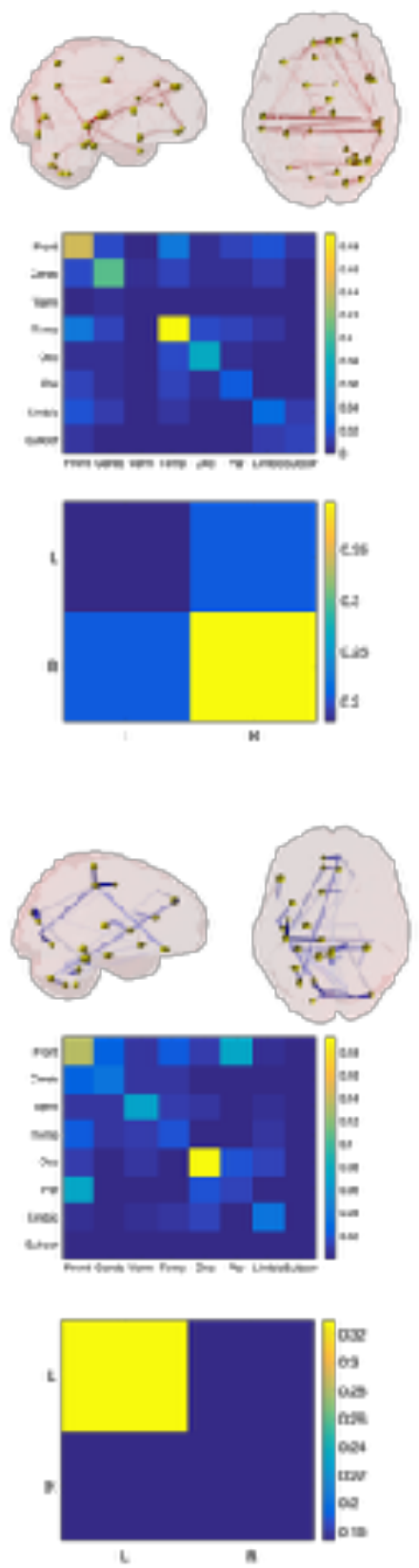

NMI
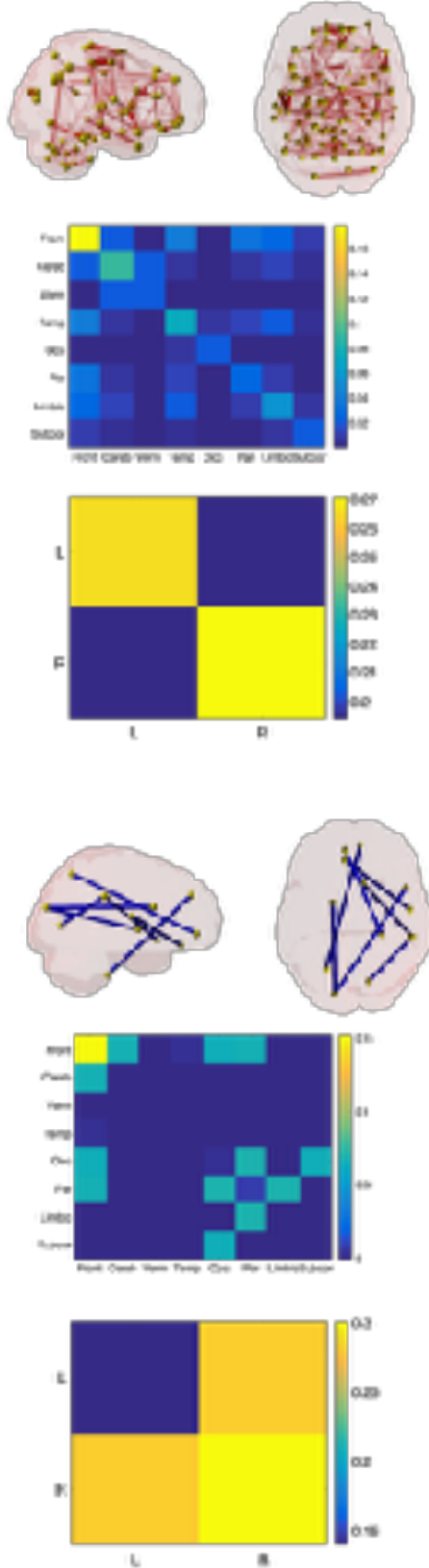

Figure 12: A visualization of the normative pathways that appear uniquely in each group. These values were obtained by subtracting the Jaccard Edge Indices in each group from each other and comparing those values with differences found in a set of null models, to determine which were statistically significant. Edge intensity in the visualization is associated with that edge's use in the selected normative path in its respective group. Matrices show the fractions of edges in each extrema that connect different regions and halves of the brain. 


\subsection{Cross-group comparisons}

Using the cross-group normative pathway comparison, we performed a groupwise comparison between the control and MDD groups, finding the normative pathways that were more frequently present in one group relative to the other, across all three modalities. Figure 9 displays the edges most used by normative pathways that were significantly different between the test group and the MDD group. In the section below, we generalize those differences.

In all three modalities, we identified, in both groups, unique normative pathways in the frontal lobe; the MDD group had unique normative pathways in the cerebellum. Normative pathways derived from correlation and partial correlation were found more utilized in the control group in the occipital lobe. The MDD group was found to utilize a number of normative pathways more in the temporal lobe. With the exception of normalized mutual information in the control group, these normative pathways were typically local in nature, occurring within brain regions and within particular lobes.

Table 1 shows the brain areas connected to the most edges in each group and their associated network found to be disrupted in depression in a meta-analysis of studies with adult participants Kaiser et al. (2015).

In the case of partial correlation, the clearest disrupted network intersected the occipital lobe, which may be related to anxiety (Goddard et al., 2001; Adenauer et al., 2010; Brühl et al., 2011; Graham et al., 2013) in patients with MDD. The right cuneus and superior and mid occipital lobe were also those three areas found in Kaiser et al. (2015) to have significant hypoconnectivity in MDD with the ventral attention network. Differential normative pathways derived from partial correlation also implicated areas previously found to be hyperconnected with the default mode network and hypoconnected to the affective network in MDD, largely validating many of the findings in Kaiser et al. (2015).

Normalized mutual information and correlation detected many new and disrupted normative pathways that intersected the cerebellum. Though the cerebellum is not implicated in the meta-analysis performed by Kaiser et al. (2015), which used a multikernel density analysis, it was found to have significantly altered connectivity in rs-fcMRI in Guo et al. (2015), a study which used Pearson correlations as the estimate of connectivity, and altered negative correlations Cao et al. (2012).

\section{Discussion and Future Work}

The overarching goal of this study was to find evidence of pathways that are utilized by the functional connectome in order to discover common, potentially underlying routes of information transfer in human brains. Our specific objective in this study was to find and analyze a consistent set of strong pathways in the functional connectome, to distinguish them from the shortest pathways, and to analyze the ways in which these paths differed between groups. This study provides evidence that these normative pathways are present in the functional connectome and utilized in different ways in MDD and control adolescents.

\subsection{Semimetricity}

The extensive development and application of graph theory in a wide range of scientific fields has encouraged its use in brain connectomics. A key concept frequently adopted is the idea of shortest pathways connecting spatially distinct regions along which information might preferentially flow. Although few studies have analyzed the application of pathfinding algorithms directly in functional connectomes, such algorithms are often used indirectly; for instance, deriving system-level structures such as "rich clubs" (van den Heuvel and Sporns, 2011), and in the calculations of metrics that characterize overall network topology, such as betweenness centrality, closeness centrality (Zuo et al., 2011), and efficiency (van den Heuvel et al., 2009). Though the idea of shortest pathways is embedded in the analysis of brain connectomes, previous studies have neither asked where these shortest pathways travel through in the brain, nor whether these pathways vary from one individual to another. Many studies have applied them to binarized functional connectomes (Bassett and Bullmore, 2006; Sporns et al., 2007; Wang et al., 2009; Lynall et al., 2010), but this approach reduces the amount of data represented by a connectome. By mapping connectomes from a proximity space to a distance 
space, we can apply pathfinding algorithms to weighted functional connectomes, giving us a richer analysis of the data.

\subsection{Normative Pathways}

As in other real-world networks, information does not necessarily travel along the shortest pathways (Borgatti, 2005; Hromkovic et al., 2005; da Fontoura Costa and Travieso, 2007). We argue against the preeminence of shortest pathways in brain connectivity and suggest instead that normative pathways are a key element to the distribution of information across the connectome. This article demonstrates that normative pathways are distinct from shortest pathways in the functional connectome; that inter hemispheric normative pathways closely follow direct callosal connections (Figure 9), which, considering previous work showing that interhemispheric functional connections are closely related to the integrity of the corpus callosum (Quigley et al., 2003; Johnston et al., 2008; Putnam et al., 2008; Uddin et al., 2008), suggests that they may follow the underlying biological substrate; and that analysis of their presence can yield knowledge about the differences between subnetworks in patient groups. Additionally, our random matrix simulations and single-group normative pathway analysis suggest that different modalities may reveal different properties and effects in the underlying data, if they are present. This is supported by our cross-group comparison of normative pathways, which generally yielded different results depending on the modalities used, but revealed different subnetworks that were consistent with previous literature.

Studies in functional connectivity that are concerned with the analysis of the connectome itself (rather than methods of deriving the connectome from raw fMRI data, which this article is largely unconcerned with) are often concerned with describing the general structure of the connectome (e.g. the small-world hypothesis (Bassett and Bullmore, 2006; Sporns, 2006; Salvador et al., 2005; Achard et al., 2006)), community partitions, or finding subnetworks such as the default mode(He et al., 2009; Smith et al., 2009; Betzel et al., 2016; Sporns and Betzel, 2016; Nicolini et al., 2017); or centrality, such as finding which parts of the brain play a central (i.e., more important) role in network dynamics(Sporns et al., 2007; Joyce et al., 2010; Zuo et al., 2011). However, initial work on functional pathways in the brain was limited due to the use of binarized networks (Bassett and Bullmore, 2006; Sporns et al., 2007; Wang et al., 2009; Lynall et al., 2010).

Avena-Koenigsberger et al. (2017) preceded this article in the use of Yen's $k$ shortest path algorithm, arguing against the importance of shortest pathways in connectivity by analysing path ensembles between brain regions in individual structural connectomes, relaxing the assumption that a shortest path must be taken. Our method, by selecting one common path among a group of participants, addresses stability and reproducibility problems unique to rsfMRI (Honey et al., 2009). While our methods do not exclude the hypothesis that signal communication may occur over an ensemble of pathways, they are more concerned with finding whether at least one viable pathway exists in the unstable topology of fMRI connectomes.

In this study, we conducted several different tests concerning the composition of the normative pathways. The inverse relationship between the average efficiency and the global Jaccard Edge Index (seen in Figures 3 and 4 ), and the use of lower-weighted, but statistically significant edges at $K=20$ (Figure 9) were largely predictable results, but were used to understand the properties of the Jaccard Edge Index Maximization Algorithm under controlled conditions, and to compare these values across different modalities. The most surprising aspect of this analysis was the differing variances in closeness centrality between the test $(N=34)$ and the retest $(N=30)$ groups; specifically, the variance increased between $K=[1 \ldots 20]$ for the test group but decreased for the retest group.

We found that normalized mutual information had the highest Global Jaccard Edge Index, correlation had the second highest, and partial correlation the lowest. This could mean that normalized mutual information naturally produces more stable pathways in its topology, or it means that other factors, such as average path length and degree distribution, trivially lower the Global Jaccard Edge Index. The latter reason is most likely the case. In the test group, normalized mutual information's average normative path length was smaller than that associated with other modalities (2.12 for normalized mutual information versus 3.48 and 3.93 for Pearson's correlation and partial correlation, respectively). As there are fewer possible one- or two-edged pathways that may connect two nodes, it is more likely that the Jaccard Edge Index Maximization Algorithm would converge on one of these as a normative pathway, thus raising the Global Jaccard Edge Index. Likewise, a lower average degree distribution of edge lengths (as normalized mutual information displays; see the histograms in Figure 9) in proximity space would inflate edge values in distance space (after application of Equation 1), 
favouring the use of fewer edges in pathways. Within modalities, the degree distributions of correlation and partial correlation were similar, with the normative pathways using a wider variety of edges than their counterpart shortest pathways (see Figures 6 and 11).

In the within-group analyses of the controls, the greatest evidence of the importance of the normative pathways is the greater use of edges along the cingulum, striatum, and the upper cerebellum, which we would expect to be a natural bottleneck of structural paths connecting the cerebral hemispheres and the cerebellum (this is particularly true of partial correlation, which is discussed below). This supports the idea that the functional connectome is constrained by major white matter pathways, and that normative paths consisting of a larger number of edges are able to be visualized as following these constraints more closely than shortest pathways (Figure 9).

\subsection{Modality differences}

An interesting question to address is why modalities behaved in such different ways in these analyses. It is common practice in connectivity studies to select a favoured modality without considering other possibilities. This is a likely reason for some discrepancies in findings between different studies in rs-fcMRI, that can influence subsequent meta-analyses. Connectivity measures include Pearson's correlation(Eguiluz et al., 2005; Buckner et al., 2009; He et al., 2009; Wang et al., 2009), partial correlation(Liu et al., 2008; Nakamura et al., 2009; Zhang et al., 2011), and mutual information(Salvador et al., 2008; Lynall et al., 2010; Eqlimi et al., 2013), as well as coherence(Bassett and Bullmore, 2006; Bassett et al., 2013), wavelet-based methods(Lynall et al., 2010), and other original methods that explore relationships in the frequency domain (Salvador et al., 2008; Goelman et al., 2017). Different types of analyses may also produce different results; while Kaiser et al. (2015) used a multilevel kernel density analysis, for instance, Mulders et al. (2015) looked at studies that used both a seed-based correlation analysis and independent component analysis.

We offer an explanation that partial correlation, by regressing out the global signal, is more focal in nature, and that correlation and normalized mutual information are more suited to detect global normative pathways and disruptions. Through fMRI simulations, Smith et al. (2011) found partial correlation to be among the most effective measures of connectivity, as it correctly detected connections in simulated data at a higher rate than most other modalities tested. Our findings, likewise, support the efficacy of partial correlation in three contexts.

First, in our ground-truth simulations, partial correlation for locally-seeded edges detected seeded pathways more effectively than any other measurement (Figure 11).

Second, the within-group analysis revealed a higher path usage that included the cingulum and other callosal areas bridging the cerebral hemispheres (Figure 9), while the cross-group analysis implicated many areas in the middle of the cerebellum and the corpus callosum. Previous studies have shown that the integrity of the corpus callosum is related to inter hemispheric resting-state functional connectivity (Quigley et al., 2003; Johnston et al., 2008; Putnam et al., 2008; Uddin et al., 2008). If white matter pathways bottleneck between the left and right brain in the corpus callosum, then the increased usage and emphasis of those areas is evidence that the normative pathways follow an underlying anatomical substrate that intersects these areas more frequently than the shortest pathways.

The last place that supports the strength of partial correlation is in the cross-group analysis, in which more areas previously implicated in MDD-control group differences in Kaiser et al. (2015) were found by partial correlation than the other two other modalities (Table 3). The Kaiser et al. (2015) analysis, however, considered older age groups, so our analysis may only have found the areas implicated in the early stages of depression.

While this indicates that partial correlation is a particularly effective means of modelling the data, we view correlation and normalized mutual information as simply alternate means of modelling the data. While partial correlation showed a clear dominance of areas adjacent to the corpus callosum, normalized mutual information also had a strong increase in usage of the left and right middle cinguli, while correlation showed increased use of edges in areas that connected either halves of the cerebellum to the rest of the brain (Figure 9).

When making a practical choice of which modality to use, we would generally recommend the use of partial correlation for the above reasons. Nonetheless, Pearson's correlation remains the more prevalent metric of connectivity, and it's use allows easier comparison a wider variety of other studiest. Furthermore, partial 
correlation may be impractical on finer parcellations, or on datasets with fewer timepoints, since the number of time points cannot exceed the number of nodes in the parcellation. Normalized mutual information is advantageous $\sin$ avoiding the negative edge problem.

\subsection{Case-control differences in depression}

Many different methods have been developed to analyze functional connectivity (Li et al., 2009). This and other studies have found many different approaches of finding groupwise differences in brain images, and these different methods often offer different results. MDD has been studied extensively(Zhang et al., 2011; Bora et al., 2013; Graham et al., 2013; Li et al., 2013; Roiser and Sahakian, 2013; Singh and Gotlib, 2014; Qiu et al., 2015). Using different methodologies, different studies and meta-analyses have implicated case-control differences (both in terms of structure and function) in many different parts of the brain(Kaiser et al., 2015; Mulders et al., 2015), and others have shown only limited areas of difference (Bora et al., 2013). There are several possible explanations for this. The first is that MDD is a complex disorder and each methodology uniquely captures a different aspect of the disorder. The second is that many methods used potentially capture spurious differences in the data. The third is that MDD is a system-wide disorder and different methods implicate specific parts of the brain, each partially illuminating a deeper, more widespread effect. Another explanation for the dissimilarities is the slight differences in the datasets studied; for instance, here we studied adolescents, and so a comparison to studies on MDD in adults is not one-on-one; or, individual datasets may simply be too small to give statistically reliable results. This begs the question of whether normative pathway analysis is a comprehensive means of describing a system-wide disorder, or just another analysis method that offers its view of depression.

\subsection{Considerations in the interpretation of normative pathways}

There are several controversies surrounding the interpretation of pathways in functional connectomes, which partially stems from controversies with functional connectivity itself. First, there are functional connections that are not fully accounted for by the underlying structural connectivity (Honey et al., 2009; Meier et al., 2016), and which may not be explained by two-edged indirect pathways. Although there is evidence in time-lag-based analyses that information propogates, either directly or indirectly, across functional connections (Cole et al., 2016; Mitra and Raichle, 2016; Ito et al., 2017), there remains concern that observed causality in the BOLD signal is due to the kinetics of neurovascular coupling (Handwerker et al., 2004; Friston, 2009).

More fundamentally, the analysis of pathways in functional connectomes is complicated by the presence of relatively strong edges that may be the byproduct of the shared variance of an indirect pathway, rather than a true instance of information transfer. For instance, an indirect pathway $B->A->C$ may introduce shared variance between $B$ and $C$ by their relationships with $A$ that expresses as a strong edge $B->C$, despite the lack of any direct information transfer between regions $B$ and $C$.

There are means of calculating whether, for an indirect path, the shared variance between two areas is stronger than the calculated indirect pathway. Consider the three-node case, B->A->C. For Pearson's correlation, the following inequality holds:

If $\operatorname{corr}(A, B)=c, \operatorname{corr}(B, C)=a$, and $\operatorname{corr}(C, A)=b$, then $a>=b c-\sqrt{ }\left(1-c^{\wedge} 2\right) \sqrt{ }\left(1-b^{\wedge} 2\right)$

And, if the following is true:

$1 /(1+((1-1 / b)+(1-1 / c)))>a$,

then the indirect pathway is stronger than its shared variance, and, when calculating normative pathways, the indirect pathway would rank higher in the list of the $k$ shortest pathways than the direct edge, making it more likely that the Jaccard Edge Index Maximization Algorithm would converge on the indirect pathway.

To generalize this, of course, one must consider degree distributions (which, as we have shown, vary substantially between Pearson's correlation, partial correlation, and normalized mutual information), the transitivity qualities of the considered modality (i.e., the above equation for Pearson's correlation), the selected t-norm used to invert and sum edges, and the number of edges in a given path. 
In general, if the indirect pathway, calculated by Equation 3, is consistently stronger than the direct edge connecting two areas, it is more likely to be converged upon by the Jaccard Edge Index Maximization Algorithm and identified as a normative pathway.

\section{Conclusion}

In this study, we propose an alternative measurement to shortest pathways in weighted functional connectomes. We demonstrate that the composition of shortest pathways in functional connectomes is inconsistent and we propose a means of improving this by discovering the normative pathways. We show that the resulting pathways from this algorithm closely utilize key anatomical areas close to the corpus callosum, which have been shown to be key to inter hemispheric functional connectivity, especially when the connectome is modelled using partial correlation. We demonstrate, as well, that the areas in the functional connectome where these normative pathways converge differently in participants with MDD and controls correspond to findings in other studies of connectivity. This demonstrates the usefulness of the method. Future studies assessing the relationship between normative pathways and underlying white matter connectivity are of importance and may improve our understanding of the relationship between functional and structural connectomes.

\section{Acknowledgements}

The study was funded by the UK Medical Research Council [grant number G0802226], the National Institute for Health Research (NIHR) [grand number 06-05-01], financial support from the Department of Health, and the Behavioral and Clinical Neuroscience Institute (BCNI), University of Cambridge, the latter being jointly funded by the Medical Research Council and the Wellcome Trust. Additional support was received from the Cambridge Biomedical Research Centre. Matthew Leming was funded by a Gates Cambridge Scholarship from the University of Cambridge. We also thank the support from Alzheimer's Research UK [grand number ARUKSRF2017B-1].

Special thanks go to all participants for their contribution to this work. We also greatly appreciate the role of the Wolfson Brain Imaging Centre, Cambridgeshire and Peterborough NHS Foundation Trust, Child and Adolescent Mental Health Services, Mental Health Research Network, IMPACT research assistants, and IMPACT clinicians, without whom this study could not have taken place. 


\section{References}

- Abdelnour, F., Voss, H., and Raj, A. (2014). Network diffusion accurately models the relationship between structural and functional brain connectivity networks. Neurolmage, 90:335-347.

- Achard, S. and Bullmore, E. (2007). Efficiency and cost of economical brain functional networks. PLoS Comput Biol, 3:e17.

- Achard, S., Salvador, R., Whitcher, B., Suckling, J., and Ed Bullmore, E. (2006). A resilient, lowfrequency, small-world human brain functional network with highly connected association cortical hubs. Journal of Neuroscience, 26:63-72.

- Adenauer, H., Pinosch, S., Catani, C., Gola, H., Keil, J., Kissler, J., and Neuner, F. (2010). Early processing of threat cues in posttraumatic stress disorder-evidence for a cortical vigilance-avoidance reaction. Biological Psychiatry, 68:451-458.

- Anderson, A. and Thomason, M. (2013). Functional plasticity before the cradle: a review of neural functional imaging in the human fetus. Neurosci Biobehav Rev., 37:2220-2232.

- Anderson, V., Catroppa, C., Morse, S., Haritou, F., and Rosenfeld, J. (2005). Functional plasticity or vulnerability after early brain injury? Pediatrics, 116:1374-1382.

- Avena-Koenigsberger, A., Misic, B., Hawkins, R., Griff, A., Hagmann, P., Goni, J., and Sporns, O. (2017). Path ensembles and a tradeoff between communication efficiency and resilience in the human connectome. Brain Struct Funct, 222:603-618.

- Bassett, D. and Bullmore, E. (2006). Small-world brain networks. The Neuroscientist, 12:512-523.

- Bassett, D., Porter, M., Wymbs, N., Grafton, S., Carlson, J., and Mucha, P. (2013). Robust detection of dynamic community structure in networks. Chaos: An Interdisciplinary Journal of Nonlinear Science, 23:013142.

- Bavelas, A. (1950). Communication patterns in task-oriented groups. J. Acoust. Soc. Am, 22:725-730.

- Benjamini, Y. and Hochberg, Y. (1995). Controlling the false discovery rate: A practical and powerful approach to multiple testing. Journal of the Royal Statistical Society. Series B (Methodological), 57:289- 300 .

- Betzel, R., Avena-Koenigsberger, A., Goñi, J., He, Y., de Reus, M., Griffa, A., and van den Heuvel, M. (2016). Generative models of the human connectome. Neurolmage, 124:1054-1064.

- Betzel, R., Byrge, L., He, Y., Goñi, J., Zuo, X., and Sporns, O. (2014). Changes in structural and functional connectivity among resting-state networks across the human lifespan. Neurolmage, 102:345-357.

- Boguña, M., Krioukov, D., and Claffy, K. (2009). Navigability of complex networks. Nat Phys, 5:74-80. Bora, E., Harrison, B., Yücel, M., and Pantelis, C. (2013). Cognitive impairment in euthymic major depressive disorder. Psychol Med., 43:2017-2026.

- Borgatti, S. (2005). Centrality and network flow. Social Networks, 27:55-71.

- Brühl, A., Rufer, M., Delsignore, A., Kaffenberger, T., Jancke, L., and Herwig, U. (2011). Neural correlates of altered general emotion processing in social anxiety disorder. Brain Research, 1378:7283.

- Buckner, R., Sepulcre, J., Talukdar, T., Krienen, F., Liu, H., Hedden, T., Andrews-Hanna, J., Sperling, R., and Johnson, K. (2009). Cortical hubs revealed by intrinsic functional connectivity: mapping, assessment of stability and relation to alzheimer's disease. J. Neurosci., 29:1860-1873.

- Buzsaki, G., Geisler, C., Henze, D., and Wang, X. (2004). Interneuron diversity series: Circuit complexity and axon wiring economy of cortical interneurons. Trends Neurosci, 27:186-193.

- Cao, M., Wang, J., Dai, Z., Cao, X., Jiang, L., Fan, F., Song, X., Xia, M., Shu, N., Dong, Q., Milham, M., Castellanos, F., Zuo, X., and He, Y. (2014). Topological organization of the human brain functional connectome across the lifespan. Dev Cogn Neurosci., 7:76-93.

- Cao, X., Liu, Z., Xu, C., Li, J., Gao, Q., Sun, N., Xu, Y., Ren, Y., Yang, C., and Zhang, K. (2012). Disrupted resting-state functional connectivity of the hippocampus in medication-na"ıve patients with major depressive disorder. Journal of Affective Disorders, 141:194-203.

- Chattopadhyay, S., Tait, R., Simas, T., Nieuwenhuizen, A., Hagan, C., Holt, R., Graham, J., Sahakian, B., Wilkinson, P., Goodyer, I., and Suckling, J. (2017). Cognitive behavioral therapy lowers elevated functional connectivity in depressed adolescents. EBioMedicine, 17:216-222.

- Cole, M., Yang, G., Murray, J., Repov`s, G., and Anticevic, A. (2016). Functional connectivity change as shared signal dynamics. J Neurosci Methods, 259:22-39.

- Cormen, T., Leiserson, C., Rivest, R., and Stein, C. (2009). Introduction to Algorithms. Massachusetts Institute of Technology, Cambridge, Massachusetts, 3rd edition. 
- da Fontoura Costa, L. and Travieso, G. (2007). Exploring complex networks through random walks. Phys Rev E, 75:016102.

- Delbeuck, X., van der Linden, M., and Collette, F. (2003). Alzheimer's disease as a disconnection syndrome? Neuropsychology, 13:79-92.

- Dijkstra, E. (1959). A note on two problems in connection with graphs. Numerische Mathematik, 1:269271. Dombi, J. (1982). A general class of fuzzy operators, the demorgan class of fuzzy operators and fuzziness measures induced by fuzzy operators. Fuzzy Sets and Systems, 8:149-163.

- Eguiluz, V., Chialvo, D., Cecchi, G., Baliki, M., and Apkarian, A. (2005). Scale-free brain functional networks. Phys Rev Lett, 94:018102.

- Eqlimi, E., Riyahi Alam, N., Sahraian, M., Eshaghi, A., Riyahi Alam, S., Ghanaati, H., Firouznia, K., and Karami, E. (2013). Resting state functional connectivity analysis of multiple sclerosis and neuromyelitis optica using graph theory. XIII Mediterranean Conference on Medical and Biological Engineering and Computing 2013, 41:206-209.

- Estrada, E. and Hatano, N. (2008). Communicability in complex networks. Phys Rev E, 77:036111. Fox, M., Zhang, D., Snyder, A., and Raichle, M. (2009). The global signal and observed anticorrelated resting state brain networks. Journal of Neurophysiology, 101:3270-3283.

- Freeman, L. (1977). A set of measures of centrality based on betweenness. Sociometry, 40:35-41.

- Freeman, L. (1979). Centrality in social networks: Conceptual clarification. Social Networks, 1:215-239.

- Friston, K. (2009). Causal modelling and brain connectivity in functional magnetic resonance imaging. PLoS Biol, 7:e1000033.

- Friston, K., Frith, C., Little, P., and Frackowiak, R. (1993). Functional connectivity: The principalcomponent analysis of large (pet) data sets. Journal of Cerebral Blood Flow and Metabolism, 33:514.

- Galán, R. (2008). On how network architecture determines the dominant patterns of spontaneous neural activity. PLoS One, 3:e2148.

- Goddard, A., Mason, G., Almai, A., Rothman, D., Behar, K., Petroff, O., Charney, D., and Krystal, J. (2001). Reductions in occipital cortex gaba levels in panic disorder detected with h-1-magnetic resonance spectroscopy. Archives of General Psychiatry, 58:556-561.

- Goelman, G., Dan, R., Rǔzi`cka, F., Bezdicek, O., Ru`zǐcka, E., Roth, J., Vymazal, J., and Jech, R. (2017). Frequency-phase analysis of resting-state functional mri. Nature Scientific Reports, 7.

- Gong, G., Rosa-Neto, P., Carbonell, F., Chen, Z., He, Y., and Evans, A. (2009). Age-and gender-related differences in the cortical anatomical. J Neurosci, 29:15684-15693.

- Goñi, J., Avena-Koenigsberger, A., de Mendizabal, N., Heuvel, M., Betzel, R., and Sporns, O. (2013a). Exploring the morphospace of communication efficiency in complex networks. PLoS One, 8:e58070.

- Goñi, J., Heuvel, M., Avena-Koenigsberger, A., Mendizabal, N., Betzel, R., Griffa, A., Hagmann, P., Corominas-Murtra, B., Thiran, J., and Sporns, O. (2013b). Resting-brain functional connectivity predicted by analytic measures of network communication. PNAS, 111:833-838.

- Graham, J., Salimi-Khorshidi, G., Hagan, C., Walsh, N., Goodyer, I., Lennox, B., and Suckling, J. (2013). Meta-analytic evidence for neuroimaging models of depression: state or trait? J Affect Disord., 151:423- 431.

- Greenwood, P. (2007). Functional plasticity in cognitive aging: review and hypothesis. Neuropsychology, 21:657-673.

- Guimera, R., Sales-Pardo, M., and Amaral, L. (2007). Classes of complex networks defined by role-torole connectivity profiles. Nat Phys, 3:63-69.

- Guo, W., Liu, F., Liu, J., Yu, M., Zhang, Z., Liu, G., Xiao, C., and Zhao, J. (2015). Increased cerebellardefault-mode-network connectivity in drug-naive major depressive disorder at rest. Medicine (Baltimore), 94:e560.

- Hagan, C., Graham, J., Tait, R., Widmer, B., van Nieuwenhuizen, A., Ooi, C., Whitaker, K., Simas, T., Bullmore, E., Lennox, B., Sahakian, B., Goodyer, I., and Suckling, J. (2015). Adolescents with current major depressive disorder show dissimilar patterns of age-related differences in acc and thalamus. Neuroimage: Clinical, 7:391-399.

- Hagan, C., Graham, J., Widmer, B., Holt, R., Ooi, C., van Nieuwenhuizen, A., Fonagy, P., Reynolds, S., Target, M., Kelvin, R., Wilkinson, P., Bullmore, E., Lennox, B., Sahakian, B., Goodyer, I., and Suckling, J. (2013). Magnetic resonance imaging of a randomized controlled trial investigating predictors of recovery following psychological treatment in adolescents with moderate to severe unipolar depression: study protocol for magnetic resonance-improving mood with psychoanalytic and cognitive therapies (mr- impact). BMC Psychiatry, 13. 
- Hagmann, P., Cammoun, L., Gigandet, X., Meuli, R., Honey, C., Wedeen, V., and Sporns, O. (2008). Mapping the structural core of human cerebral cortex. PLoS Biol, 6:e159.

- Handwerker, D., Ollinger, J., and D'Esposito, M. (2004). Variation of bold hemodynamic responses across subjects and brain regions and their effects on statistical analyses. Neurolmage, 21:16391651.

- He, Y., Wang, J., Wang, L., Chen, Z., Yan, C., Yang, H., Tang, H., Zhu, C., Gong, Q., Zang, Y., and Evans, A. (2009). Uncovering intrinsic modular organization of spontaneous brain activity in humans. PLoS One, 4:e5226.

- Hermundstad, A., Bassett, D., Brown, K., Aminoff, E., Clewett, D., Freeman, S., Frithsen, A., Johnson, A., Tipper, C., Miller, M., Grafton, S., and Carlson, J. (2013). Structural foundations of resting-state and task-based functional connectivity in the human brain. PNAS, 110:6169-6174.

- Hilgetag, C., Burns, G., O'Neill, M., Scannell, J., and Young, M. (2000). Anatomical connectivity defines the organisation of cortical areas in the macaque monkey and the cat. Phil. Trans. R. Soc. Lond., 355:91-110.

- Honey, C., Sporns, O., Cammoun, L., Gigandet, X., Thiran, J., Meuli, R., and Hagmann, P. (2009). Predicting human resting-state functional connectivity from structural connectivity. PNAS, 106:20352040.

- Hromkovic, J., Klasing, R., Pelc, A., Ruzicka, P., and Unger, W. (2005). Dissemination of Information in Communication Networks: Broadcasting, Gossiping, Leader Election, and Fault-Tolerance. SpringerVerlag, Berlin, Heidelberg.

- Ito, T., Kulkarni, K., Schultz, D., Mill, R., Chen, R., Solomyak, L., and Cole, M. (2017). Cognitive task infor- mation is transferred between brain regions via resting-state network topology. Nature Communications, 8:1027.

- Johnston, J., Vaishnavi, S., Smyth, M., Zhang, D., He, B., Zempel, J., Shimony, J., Snyder, A., and Raichle, M. (2008). Loss of resting interhemispheric functional connectivity after complete section of the corpus callosum. J Neurosci., 28:6453-6458.

- Joyce, K., Laurienti, P., Burdette, J., and Hayasaka, S. (2010). A new measure of centrality for brain networks. PLoS ONE, 5:e12200.

- Kaiser, M., Martin, R., Andras, P., and Young, M. (2007). Simulation of robustness against lesions of cortical networks. Eur J Neurosci, 25:3185-3192.

- Kaiser, R., Andrews-Hanna, J., Wager, T., and Pizzagalli, D. (2015). Large-scale network dysfunction in major depressive disorder: A meta-analysis of resting-state functional connectivity. JAMA Psychiatry, 72:603-611.

- Latora, V. and Marchiori, M. (2001). Efficient behavior of small-world networks. Phys. Rev. Lett., $87: 198701$. Leech, R. and Sharp, D. (2014). The role of the posterior cingulate cortex in cognition and disease. Brain,

- 137:12-32.

Li, B., Liu, L., Friston, K., Shen, H., Wang, L., Zeng, L., and Hu, D. (2013). A treatment-resistant default mode subnetwork in major depression. Biological Psychiatry, 74:48-54.

- Li, K., Guo, L., Nie, J., Li, G., and Liu, T. (2009). Review of methods for functional brain connectivity detection using fmri. Computerized medical imaging and graphics: the official journal of the Computerized Medical Imaging Society, 33:131-139.

- Liu, Y., Liang, M., Zhou, Y., He, Y., Hao, Y., Song, M., Yu, C., Liu, H., Liu, Z., and Jiang, T. (2008). Disrupted small-world networks in schizophrenia. Brain, 131:945-961.

- Lohmann, G., Margulies, D., Horstmann, A., Pleger, B., Lepsien, J., Goldhahn, D., H., S., Stumvoll, M., Villringer, A., and Turner, R. (2010). Eigenvector centrality mapping for analyzing connectivity patterns in fmri data of the human brain. PLoS ONE, 5:e10232.

- Lynall, M., Bassett, D., Kerwin, R., McKenna, P., Kitzbichler, M., Muller, U., and Bullmore, E. (2010). Functional connectivity and brain networks in schizophrenia. J Neurosci, 30:9477-9487.

- Masuda, N. and Aihara, K. (2004). Global and local synchrony of coupled neurons in small-world networks. Biol. Cybern, 90:302-309.

- Meier, J., Topka, M., and H" anggi, J. (2016). Differences in cortical representation and structural connectivity of hands and feet between professional handball players and ballet dancers. Neural Plast., 2016.

- Milgram, S. (1967). The small-world problem. Psychology Today, 1:61-67. Mitra, A. and Raichle, M. (2016). How networks communicate: propagation patterns in spontaneous brain 
- activity. Philos Trans R Soc Lond B Biol Sci., 371:20150546.

Mi`sí c, B., Betzel, R., Nematzadeh, A., Goñi, J., Griffa, A., Hagmann, P., and Sporns, O. (2015). Cooperative and competitive spreading dynamics on the human connectome. Neuron, 86:15181529. 25

- Mucha, P., Richardson, T., Macon, K., Mason, A. Porter, M., and Onnela, J. (2010). Community structure in time-dependent, multiscale, and multiplex networks. Science, 328:876-878.

- Mulders, P., van Eijndhoven, P., Schene, A., Beckmann, C., and Tendolkar, I. (2015). Resting-state functional connectivity in major depressive disorder: A review. Neurosci Biobehav Rev., 56:330-344.

- Murphy, K., Birn, R., Handwerker, D., Jones, T., and Bandettini, P. (2009). The impact of global signal regression on resting state correlations: are anti-correlated networks introduced? Neurolmage, 44:893-905.

- Nakamura, T., Hillary, F., and Biswal, B. (2009). Resting network plasticity following brain injury. PLoS One, 4:e8220.

- Nicolini, C., Bordier, C., and Bifone, A. (2017). Community detection in weighted brain connectivity networks beyond the resolution limit. Neurolmage, 146:28-39.

- Park, H. and Friston, K. (2013). Structural and functional brain networks: from connections to cognition. Science, 342:1238411.

- Passingham, R., Stephan, K., and K"otter, R. (2002). The anatomical basis of functional localization in the cortex. Nat. Rev. Neurosci., 3:606-616.

- Putnam, M., Wig, G., Grafton, S., Kelley, W., and Gazzaniga, M. (2008). Structural organization of the corpus callosum predicts the extent and impact of cortical activity in the nondominant hemisphere. $J$ Neurosci., 28:2912-2918.

- Qiu, A., Anh, T., Rifkin-Graboi, A., Broekman, B., Kwek, K., Saw, S., Chong, Y., Gluckman, P., Fortier, N., and Meaney, M. (2015). Prenatal maternal depression alters amygdala functional connectivity on 6month-old infants. Translational Psychiatry, 5.

- Quigley, M., Cordes, D., Turski, P., Moritz, C., Haughton, V., Seth, R., and Meyerand, M. (2003). Role of the corpus callosum in functional connectivity. AJNR Am J Neuroradiol, 24:208-212.

- Roberts, B. and Kroese, D. (2007). Estimating the number of s-t paths in a graph. Journal of Graph Algorithms and Applications, 11:195-214.

- Rocha, L. (2002). Proximity and semi-metric analysis of social networks: Advanced knowledge integration in assessing terrorist threats. Technical Report LAUR 02-6557, Los Aalamos National Laboratory, Los Alamos, New Mexico.

- Roiser, J. and Sahakian, B. (2013). Hot and cold cognition in depression. CNS Spectr., 18:139-149. Rubinov, M. and Sporns, O. (2010). Complex network measures of brain connectivity: Uses and interpretations. Neurolmage, 52:1059-1069.

- Salvador, R., Martinez, A., Pomarol-Clotet, E., Gomar, J., Vila, F., Sarro, S., Capdevila, A., and Bullmore, E. (2008). A simple view of the brain through a frequency-specific functional connectivity measure. Neuroimage, 39:279-289.

- Salvador, R., Suckling, J., Schwarzbauer, C., and Bullmore, E. (2005). Undirected graphs of frequencydependent functional connectivity in whole brain networks. Philos Trans R Soc Lond B Biol Sci, 360:937-946.

- Serrano, M., Maguitman, A., Boguna, M., Fortunato, S., and Vespignani, A. (2007). Decoding the structure of the www: A comparative analysis of web crawls. ACM Transactions on the Web, 1.

- Simas, T. (2012). Stochastic Models and Transitivity in Complex Networks. PhD dissertation, Indiana University.

- Simas, T., Chattopadhyay, S., Hagan, C., Kundu, P., Patel, A., Holt, R., Floris, D., Graham, J., Ooi, C., Tait, R., Spencer, M., Baron-Cohen, S., Sahakian, B., Bullmore, E., Goodyer, I., and Suckling, J. (2015). Semi-metric topology of the human connectome: Sensitivity and specificity to autism and major depressive disorder. PLoS One, 10.

- Simas, T. and Rocha, L. (2014). Distance closures on complex networks. Singh, M. and Gotlib, I. (2014). The neuroscience of depression: Implications for assessment and intervention. Behav Res Ther., 62:60-73.

- Smith, S., Fox, P., Miller, K., Glahn, D., Fox, P., Mackay, C., Filippini, N., Watkins, K., Toro, R., Laird, A., and Beckmann, C. (2009). Correspondence of the brain's functional architecture during activation and rest. Proc. Natl. Acad. Sci., 106:13040-13045.

- Smith, S., Miller, K., Salimi-Khorshidi, G., Webster, M., Beckmann, C., Nichols, T., Ramsey, J., and Woolrich, M. (2011). Network modelling methods for fmri. Neurolmage, 54:875-891. 
- Sporns, O. (2006). Small-world connectivity, motif composition, and complexity of fractal neuronal connec- tions. BioSystems, 85:55-64.

- Sporns, O. and Betzel, R. (2016). Modular brain networks. Annu Rev Psychol., 67:613-640.

Sporns, O., Honey, C., and K" otter, R. (2007). Identification and classification of hubs in brain networks. PLoS One, 2:e1049.

Sporns, O., Tononi, G., and Edelman, G. (2000). Theoretical neuroanatomy: Relating anatomical and functional connectivity in graphs and cortical connection matrices. Cerebral Cortex, 10:127-141.

- Sporns, O. and Zwi, J. (2004). The small world of the cerebral cortex. Neuroinformatics, 2:145-162.

- Stephan, K., Hilgetag, C., Burns, G., O’Neill, M., Young, M., and K"otter, R. (2000). Computational analysis of functional connectivity between areas of primate cerebral cortex. Philos Trans $\mathrm{R}$ Soc Lond B Biol Sci, 355:111-126.

- Suckling, J., Simas, T., Chattopadhyay, S., Tait, R., Su, L., Williams, G., Rowe, J., and O'Brien, J. (2015). A winding road alzheimers disease increases circuitous functional connectivity pathways. Frontiers in Computational Neuroscience, 9.

- Tomasi, D. and Volkow, N. (2010). Functional connectivity density mapping. Proc. Natl. Acad. Sci., 107:9885-9890.

- Tomasi, D. and Volkow, N. (2011). Association between functional connectivity hubs and brain networks. Cereb Cortex, 21:2003-2013.

- Traag, V. and Bruggeman, J. (2009). Community detection in networks with positive and negative links. Phys. Rev. E., 80:036115.

- Tzourio-Mazoyer, N., Landeau, B., Papathanassiou, D., Crivello, F., Etard, O., Delcroix, N., Mazoyer, B., and Joliot, M. (2002). Automated anatomical labeling of activations in spm using a macroscopic anatomical parcellation of the mni mri single-subject brain. Neurolmage, 15:273-289.

- Uddin, L., Mooshagian, E., Zaidel, E., Scheres, A., Margulies, D., Clare Kelly, A., Shehzad, Z., Adelstein, J., Castellanos, F., Biswal, B., and Milham, M. (2008). Residual functional connectivity in the splitbrain revealed with resting-state fmri. Neuroreport, 19:703-709.

- van den Heuvel, M., Kahn, R., Goñi, J., and Sporns, O. (2012). High-cost, high-capacity backbone for global brain communication. PNAS, 109:11372-11377.

- van den Heuvel, M. and Sporns, O. (2011). Rich-club organization of the human connectome. Journal of Neuroscience, 31:15775-15786.

- van den Heuvel, M., Stam, C., Kahn, R., and Pol, H. (2009). Efficiency of functional brain networks and intellectual performance. J Neurosci, 29:7619-7624.

- Wang, J., Wang, L., Zang, Y., Yang, H., Tang, H., Gong, Q., Chen, Z., Zhu, C., and He, Y. (2009). Parcellation-dependent small-world brain functional networks: A resting-state fmri study. Human Brain Mapping, 30:1511-1523.

-Watts, D. and Strogatz, S. (1998). Collective dynamics of 'small-world' networks. Letters to Nature, 393:440- 442.

- Yan, C., Gong, G., Wang, J., Wang, D., Liu, D., Zhu, C., and He, Y. (2011). Sex- and brain size-related small- world structural cortical networks in young adults: a dti tractography study. Cereb Cortex, 21:449-458.

- Yen, J. (1971). Finding the k shortest loopless paths in a network. Management Science, 17:712-716. Yoo, S., Han, C., Shin, J., Seo, S., Na, D., Kaiser, M., and Seong, J. (2015). A network flow-based analysis of cognitive reserve in normal ageing and alzheimer's disease. Scientific reports, 5:10057. Zhang, J., Wang, J., Wu, Q., Kuang, W., Huang, X., and Gong, Q. (2011). Disrupted brain connectivity networks in drug-naive, first-episode major depressive disorder. Biological Psychiatry, 70:334-342.

- Zuo, X., Ehmke, R., Mennes, M., Imperati, D., Castellanos, F., Sporns, O., and Milham, M. (2011). Network centrality in the human functional connectome. Cerebral Cortex, 22:1862-1875. 


\section{Appendices}

\section{A Necessity for edge inversions in pathfinding on proximity graphs}

In order to perform path finding in proximity space, it must be inverted from proximity space to distance space (Rocha, 2002; Simas and Rocha, 2014). The shortest path problem consists of finding the shortest path between two nodes in a graph; many algorithms, such as Dijkstra's algorithm (Dijkstra, 1959; Cormen et al., 2009), have been designed to solve this problem. However, the shortest path problem, pursuant to its name, is a minimization problem. It can be applied to a binary graph by minimizing the number of edges connecting two nodes (Sporns et al., 2000; Stephan et al., 2000; Bassett and Bullmore, 2006; van den Heuvel et al., 2009), and it can be applied to a distance graph by minimizing the sum of the edges' values (Rocha, 2002; Simas and Rocha, 2014; Simas et al., 2015; Suckling et al., 2015). However, it cannot be applied to a weighted graph because the stronger edges have higher numerical values; thus, minimizing the sum of edge values would lead to a weak pathway; maximizing the sum would turn the shortest path problem into a form of the longest path problem; and minimizing the number of edges would not necessarily be the shortest path. The output, in all cases, is meaningless for our purposes. The only way to apply a shortest path algorithm to a proximity graph, therefore, is to invert its edge values and turn it into a distance graph.

\section{B Participants and MRI Data}

BOLD-sensitive MRI were acquired on a Siemens 3T Tim Trio scanner located at the Wolfson Brain Imaging Centre, University of Cambridge, UK whilst participants were resting with eyes closed on datasets from the MR-IMPACT study (Hagan et al., 2013, 2015). Details of the MRI acquisition parameters as well as explanations for participant exclusions can be found in Chattopadhyay et al. (2017). Participants and their families gave written and informed consent, and ethical approval was provided by the Cambridgeshire Research Ethics Committee (Reference: 09-H0308-168).

The control data were taken from a sample of 34 healthy adolescents ( 7 males and 27 females, aged 12 to 18 years, mean age $=15.7$, standard deviation $=1.45$ ) with no family history of depression, who were recruited by advertisement from local schools. Forty (40) were initially recruited, with a total of 6 excluded. All of the participants were rescanned six months later as part of a longitudinal study, with four excluded.

Patients with MDD were recruited from East Anglia and North London, United Kingdom. 109 participants were reported in the MR-IMPACT study (Hagan et al., 2015), of these 108 were used in Chattopadhyay et al. (2017), with exclusions for 26 participants on the basis of head motion, psychosis, withdrawals, parcellations, dropouts, and missing data, leaving 82 (18 males and 64 females, aged between 13 and 18 years, mean $=15.6$ years, standard deviation $=1.12$ years) for inclusion in this study.

\section{Code}

Code for the computations was written in Matlab, using functions from the Matlab BGL toolbox (https:// uk.mathworks.com/matlabcentral/fileexchange/10922-matlabbgl), the Brain Connectivity Toolbox (Rubinov and Sporns, 2010), and functions in Matlab for computing the $k$ shortest paths (https://uk. mathworks.com/matlabcentral/fileexchange/32513-k-shortest-path-yen-s-algorithm) and the average mutual information (https://uk.mathworks.com/matlabcentral/fileexchange/10040-average-mutualinformat). To speed up computation times, pathways were encoded as 64-bit integers, which limited the size of the pathways to $\left\lfloor\log \left(2^{64}\right) / \log (116)\right\rfloor=9$ edges for a parcellation with 116 nodes. The computations were carried out in parallel, with each graph having its 20 shortest paths for each possible connection computed independently, followed by the Jaccard Edge Index Optimization for $1 \leq k \leq 20$. Visualization functions were all written in Matlab, with the functions for reading in relevant NIFTI parcellations drawing on the Vistasoft library (https://github.com/vistalab/vistasoft).

\section{Theoretical justification for $1 / x-1$ over $1 / x$ for path inversions}

Any three nodes $a, b, c$ with the direct correlations corr $(a, b), \operatorname{corr}(b, c), \operatorname{corr}(c, a)$ between them are subject 
to the inequality $\operatorname{corr}(a, b) \geq \operatorname{corr}(b, c)^{2}+\operatorname{corr}(c, a)^{2}-\sqrt{ }\left(1+\operatorname{corr}(b, c)^{2}\right) \times \sqrt{ }\left(1+\operatorname{corr}(c, a)^{2}\right)$. Thus, when negative correlations are set to zero, $W(P)$ is subject to two intuitive constraints with regards to the direct correlation between end nodes s and e: (1) $W(P)$ is 1 --- i.e., perfectly correlated and 0 in semimetric space --- if and only if all edge weights in $P$ are 1 , which also implies that $\operatorname{corr}(s, e)$ is $1\left(W(P)=1 \Leftrightarrow \forall i \epsilon n: P_{i}=1 \Rightarrow\right.$ $\operatorname{corr}(s, e)=1)$, and (2) $W(P)$ is zero if and only if at least one edge weight in $\mathrm{P}$ is zero, or $\infty$ in semimetric space $\left(W(P)=0 \Leftrightarrow \exists i \epsilon n: P_{i}=0\right)$.

(Note that the above does not necessarily apply to normalized mutual information, which is analyzed in the results section.)

These two constraints are important and stem from the fact that we use a t-norm $1 / x-1$ instead of a simple inverse, $1 / x$, when converting from the Pearson Correlational space to semimetric space; analysis performed using a simple inverse of the Pearson correlations (Cao et al., 2014), for example, are awed because each path has a minimum length of 1 , making the path length arbitrarily dependent on the number of edges.

\section{E Relationship between the Jaccard Edge Index and the shortest path on the mean of a group of connectomes}

Why not just average a given group of connectomes in semimetric space and find the shortest paths of the result? And what is the relationship between a shortest path in this average connectome and the paths resulting from the optimization of the Jaccard Edge Index when considering $K$ paths?

For a given connection between nodes $i$ and $j$, as the Jaccard Edge Index (Equation 7 ) approaches 1, all pathways between all $N$ graphs being considered have the same edgewise composition. This set of paths making a Jaccard Edge Index of 1 is the shortest path of the average of $N$ connectomes (i.e., when edges are averaged, such that $N$ connectomes represented by $N n x n$ matrices are averaged to a single $n \times n$ matrix). This tradeoff is illustrated in Figure E.1. The number of possible pathways connecting two nodes in a fully-connected graph increases faster than exponentially (Roberts and Kroese, 2007) with the number of nodes, so this would not be computationally feasible to compute with the Jaccard Edge Index Maximization Algorithm. Nonetheless, if the input $k$ to our problem were, hypothetically, 1 , and we did have a fully-connected graph, then the Jaccard Edge Index of the resulting path would be 1.

On the other hand, if $K=1$ (i.e., only the shortest pathways were considered), then the Jaccard Edge Index would be as low as it possibly could be, while the average path length is as small as it could be (i.e., average efficiency is optimal). Thus, as $K$ rises and more paths are considered in the Jaccard Edge Index Maximization Algorithm, there is a tradeoff between efficiency and path consistency. As Figure E.1 illustrates, however, the shortest path of the average connectome may be arbitrarily higher than the shortest local paths in certain cases.

The efficiency of connectomes when different statistical relationships (in our case, Pearson correlation, partial correlation, and normalized mutual information) are used to construct them can have very different scales. To compare the average efficiency of Pearson correlation, partial correlation, and normalized mutual information as $K$ increases and the Jaccard Edge Index is optimized, we normalize the average efficiency to the range $[0,1]$ by finding its maximum average efficiency (i.e., the efficiency when only the shortest pathways are considered) and the asymptotic minimum of the efficiency as the Jaccard Edge Index approaches 1 (i.e., all the same pathways are used), which is simply the efficiency of the averaged connectome (see Figure E.1 for a toy example). 

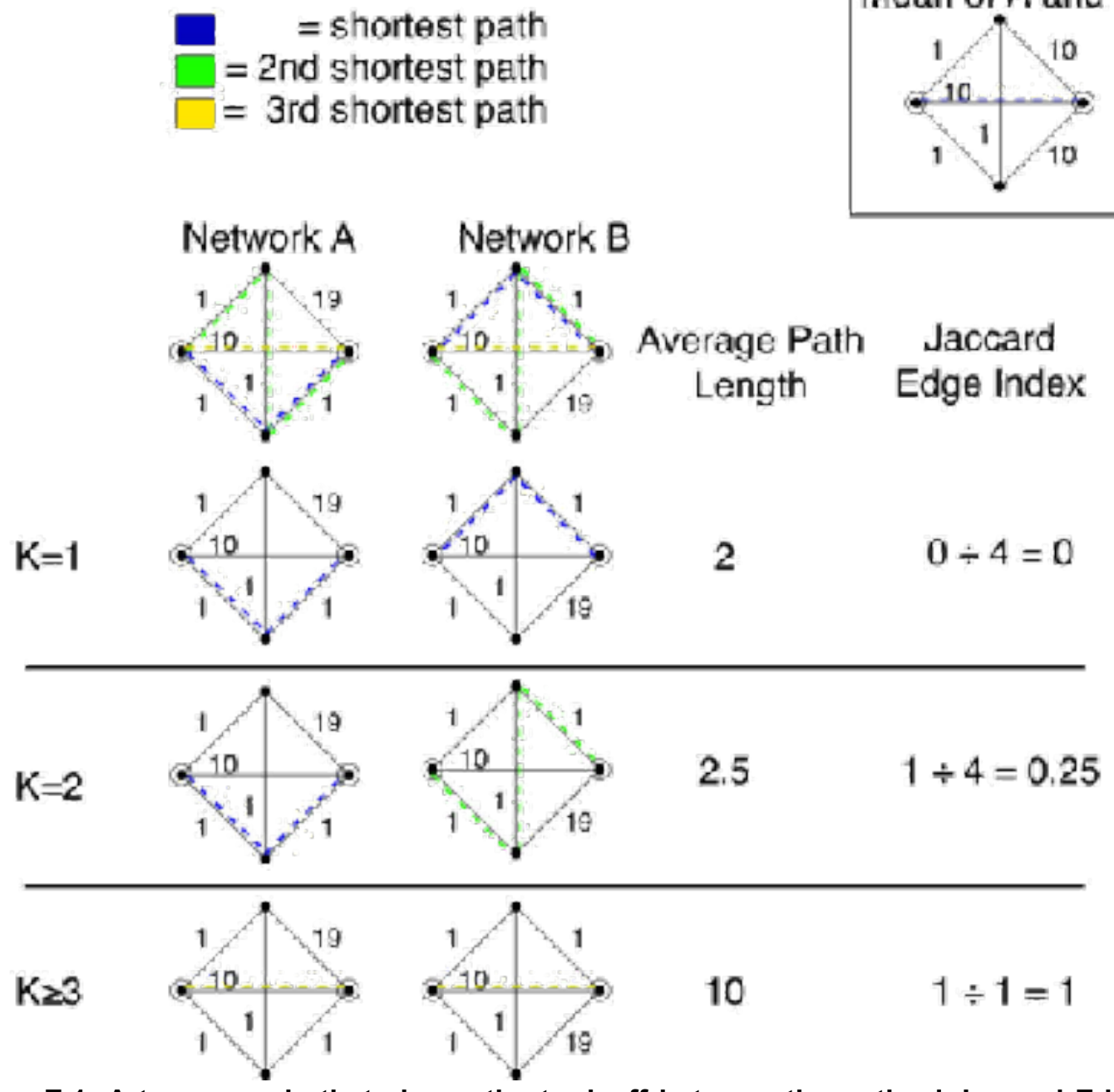

Figure E.1: A toy example that shows the tradeoff between the optimal Jaccard Edge Index and the average path length on distance graphs. In the two fully-connected networks shown, there are five possible non-looping paths that connect the circled dots; the three shortest are displayed by the dotted lines. Shown is the optimal Jaccard Edge Index, as well as the resultant average path length, that can be achieved when the $K$ shortest pathways are considered in the Optimization Algorithm (i.e., when $K=\{1,2$, $3,4,5\})$. When all pathways across networks are the same (i.e., Jaccard Edge Index equals 1), the pathway selected is the shortest pathway in the mean of all networks. This example shows that, when only the shortest pathway is considered for individual graphs, they may be different and share no common edges (the Jaccard Edge Index equals zero). Conversely, when the maximum number of possible pathways are considered (which is the same as selecting the shortest pathway of the mean of all networks), the resultant path length may be suboptimal compared with the shortest paths found in each graph locally. Thus, the Jaccard Edge Index Maximization Algorithm represents a tradeoff between consistency and efficiency, depending on the selected $K$. As the number of nodes in a graph increases, the number of possible non-looping pathways connecting two nodes grows at an extremely fast rate, so in most real-world examples it is very unlikely that a perfect Jaccard Edge Index of 1 could be achieved. 